\title{
Optical conductivity of polaronic charge carriers
}

\author{
J Loos $\dagger$, M Hohenadler $\ddagger$, A Alvermann $\S$, and H Fehske $\S$ \\ $\dagger$ Institute of Physics, Academy of Sciences of the Czech Republic, Prague \\ $\ddagger$ Theory of Condensed Matter, University of Cambridge, United Kingdom \\ $\S$ Institute of Physics, Ernst-Moritz-Arndt University Greifswald, Germany \\ E-mail: loos@fzu.cz
}

\begin{abstract}
The optical conductivity of charge carriers coupled to quantum phonons is studied in the framework of the one-dimensional spinless Holstein model. For one electron, variational diagonalisation yields exact results in the thermodynamic limit, whereas at finite carrier density analytical approximations based on previous work on single-particle spectral functions are obtained. Particular emphasis is put on deviations from weak-coupling, small-polaron or oneelectron theories occurring at intermediate coupling and/or finite carrier density. The analytical results are in surprisingly good agreement with exact data, and exhibit the characteristic polaronic excitations observed in experiments on manganites.
\end{abstract}

PACS numbers: 78.20.Bh, 71.10.-w, 71.27.+a, 75.47.Lx, 78.20.-e

Submitted to: J. Phys.: Condens. Matter

\section{Introduction}

In recent years, there has been a lot of controversy about the possible interpretation of optical data on strongly correlated electron systems such as manganites, cuprates and nickelates in terms of polaronic charge carriers $[1,2]$. It turns out that simple one-electron or perturbative weak/strong-coupling theories can usually not explain more than one type of experiment for a given set of (realistic) parameters, and a general discussion of this issue for the case of the manganites has been given in [3-5]. Owing to these discrepancies, and also in the light of recent progress in the understanding of many-polaron systems [6-8], it is highly desirable to revisit this problem using both analytical and numerical many-body methods.

Ignoring the necessity of taking into account cooperative Jahn-Teller effects to describe orbital effects in manganites [4], an important issue in the framework of one-band models concerns the validity of two complementary types of electron-phonon interaction, namely the Holstein [9] and the Fröhlich model [10] for screened, local coupling and unscreened, longrange electron-phonon interaction, respectively. Motivated by recent experimental results and the absence of a unified theory for, e.g., ferromagnetic manganites [5], we analyse the signatures of polaronic excitations in the optical conductivity, both in the low-density limit and at finite carrier density, in the framework of the one-dimensional spinless Holstein model.

Polaronic materials such as the manganites generally require a theory valid for finite carrier density and all coupling regimes [4,5]. Whereas a large number of works have reported on approximate findings for the one-electron limit (the polaron problem) of the Holstein model, here we present variational diagonalisation results for the thermodynamic 
limit, thereby providing an exact (numerical) solution. Exact data for finite clusters, also at finite temperature, can be found in [11].

The many-electron case has been studied by approximate methods in the past [3,1214]. Moreover, exact results on finite clusters are available $[15,16]$. In order to further improve the present understanding, we examine the optical conductivity in the framework of electronic spectral functions deduced by perturbative and variational methods valid at finite carrier density $[17,18]$. The important advantage of such calculations is the possibility to relate the observed contributions to the optical response to specific processes (transitions) in the analytical formulas, and to understand the relevance of these contributions in dependence on the model parameters. Special attention will be paid to the role played by the coherent respectively incoherent parts of the spectral functions. We compare the analytical results to exact numerical data, and discuss the differences between the weak-coupling (WC), intermediate-coupling (IC) and strong-coupling (SC) regimes, as well as the influence of carrier density and phonon frequency. Besides, we attempt to make a qualitative connection of our findings to recent experiments on manganites [5], as well as to theoretical work on the many-electron case based on the Holstein model [3,16] and the Fröhlich model [19,20]. Theoretical work on manganites is discussed in [3-5], and a review of polaron theories can be found in [21].

This paper is organised as follows. In section 2, we introduce the spinless Holstein model. In section 3, we outline the derivation of the general expression for the optical conductivity involving the electronic spectral functions deduced in [17]. Numerical and analytical results are discussed in section 4 , and section 5 contains our conclusions.

\section{Model}

We consider the one-dimensional (1D) spinless Holstein model, describing fermions coupled to dispersionless optical phonons. It provides a general framework to study polaron physics [22], many-polaron effects [8] and quantum phase transitions [23-26], but is simple enough to permit reliable investigations by analytical and numerical methods. A connection to the more general Holstein-Hubbard model (see, e.g., [15]) can be made in the limit of large Hubbard$U$ in the latter. Moreover, the Holstein double-exchange model for the manganites $[3,4,27]$ reduces to the present model for infinite Hund's rule coupling and a ferromagnetic state.

Following [17], we write the Hamiltonian in the general form

$$
H=\eta \sum_{i} c_{i}^{\dagger} c_{i}-\sum_{i, j} C_{i j} c_{i}^{\dagger} c_{j}+\omega_{0} \sum_{i}\left(b_{i}^{\dagger} b_{i}+\frac{1}{2}\right),
$$

where $c_{i}^{\dagger}\left(c_{i}\right)$ and $b_{i}^{\dagger}\left(b_{i}\right)$ create (annihilate) a spinless fermion respectively a phonon of energy $\omega_{0}(\hbar=1)$ at site $i$. The strength of the electron-phonon interaction is specified by the dimensionless coupling constants $\lambda=E_{\mathrm{P}} / 2 t$ (adiabatic regime, $\omega_{0} / t \ll 1$ ) and $g^{2}=E_{\mathrm{P}} / \omega_{0}$ (anti-adiabatic regime, $\omega_{0} / t \gg 1$ ), with $E_{\mathrm{P}}$ denoting the atomic-limit $\left[C_{i j}=0\right.$ for $i \neq j$ in equation (1)] polaron binding energy.

The definitions of $\eta$ and the coefficients $C_{i j}$ will depend on the type of approximation used and hence on the parameter regime. In the WC case, in which we start with the original, untransformed Holstein Hamiltonian, we have

$$
\eta=-\mu, \quad C_{i i}=g \omega_{0}\left(b_{i}^{\dagger}+b_{i}\right), \quad C_{\langle i j\rangle}=t,
$$

where $\mu$ denotes the chemical potential. In contrast, in the SC regime, we use the Hamiltonian after the Lang-Firsov transformation [28] with

$$
\eta=-E_{\mathrm{P}}-\mu, \quad C_{i i}=0, \quad C_{\langle i j\rangle}=t \mathrm{e}^{-g\left(b_{i}^{\dagger}-b_{i}-b_{j}^{\dagger}+b_{j}\right)} .
$$


The non-interacting (polaron) half-bandwidth in the SC regime is $\widetilde{W}=W \mathrm{e}^{-g^{2}}$, with the 1D free-fermion half-bandwidth $W=2 t$. Finally, the treatment of the IC case is based on a modified Lang-Firsov transformation [17] defined by the unitary operator

$$
U=\mathrm{e}^{\sum_{i} g\left(\bar{\gamma} c_{i}^{\dagger} c_{i}+\gamma\right)\left(b_{i}^{\dagger}-b_{i}\right)},
$$

which leads to a Hamiltonian of the form (1) with

$$
\begin{aligned}
& \eta=-\mu-E_{\mathrm{P}}[\bar{\gamma}(2-\bar{\gamma})+2 \gamma(1-\bar{\gamma})], \\
& C_{i i}=g \omega_{0}(1-\bar{\gamma})\left(b_{i}^{\dagger}+b_{i}\right), \quad C_{\langle i j\rangle}=t \mathrm{e}^{-\bar{\gamma} g\left(b_{i}^{\dagger}-b_{i}-b_{j}^{\dagger}+b_{j}\right)} .
\end{aligned}
$$

The parameters $\gamma$ and $\bar{\gamma}$ depend on the carrier concentration $n$, and on the variational parameter $R$ characterising the delocalisation of the charge carrier from the center of the associated lattice distortion. Explicitly, we have

$$
\gamma=2 n \mathrm{e}^{-1 / R} \tanh \frac{1}{2 R}, \quad \bar{\gamma}=\tanh \frac{1}{2 R}-\gamma,
$$

and $R$ is then defined by the position of the minimum of the total energy per site in the first (Hartree) approximation [17].

\section{Theory}

In this section we present our analytical results for the optical conductivity. We first derive a general expression which allows us to calculate the real part of the optical conductivity from the momentum and energy dependent one-electron spectral function $A(k, \omega)$. Approximations for the spectral function in different coupling regimes have been obtained in [17], and the basic formulas necessary for the computation of the conductivity are compiled in Appendix A. The calculations presented in [17] are based on the self-consistent treatment of the self-energy equations in second-order perturbation theory. To describe the crossover from the SC to the WC regime, we use the variational procedure outlined above.

Our approximation neglects vertex corrections due to direct fermion-fermion interaction, which is also absent from the spinless Hamiltonian (1). Nevertheless, fermion-phonon andat finite carrier density - phonon-mediated, retarded fermion-fermion interaction effects enter via the spectral functions [17]. For simplicity, we exclude from our discussion the quantum phase transition from a Luttinger liquid to an insulating Peierls phase at half filling [23,25].

\subsection{General expression for the optical conductivity}

The current density operator for the 1D spinless Holstein model reads

$$
\widehat{\jmath}=\mathrm{i} \frac{e t}{V} \sum_{\left\langle g^{\prime}, g\right\rangle}\left(g^{\prime}-g\right) c_{g^{\prime}}^{\dagger} c_{g},
$$

where $g^{\prime}, g$ number the lattice sites, and $a=\left|g^{\prime}-g\right|$ respectively $V=N a$ are the lattice constant and the volume.

According to linear response theory [29], the frequency dependent complex conductivity $\sigma(\omega)$ is determined by the Green function of the current density operators. In particular,

$$
\operatorname{Re} \sigma(\omega)=-\frac{V}{\omega} \operatorname{Im} G_{\widehat{\jmath}}^{\mathrm{R}}(\omega),
$$

where

$$
G_{\widehat{\jmath}}^{\mathrm{R}}(\omega)=\int_{-\infty}^{\infty} \mathrm{d}\left(t-t^{\prime}\right) G_{\widehat{\jmath}}^{\mathrm{R}}\left(t-t^{\prime}\right) \mathrm{e}^{\mathrm{i} \omega\left(t-t^{\prime}\right)}
$$


with the retarded Green function

$$
G_{\widehat{\jmath}}^{\mathrm{R}}\left(t-t^{\prime}\right)=-\mathrm{i}\left\langle\left[\widehat{\jmath}(t), \widehat{\jmath}\left(t^{\prime}\right)\right]\right\rangle \theta\left(t-t^{\prime}\right) .
$$

In the notation of [30], the related Matsubara Green function takes the form

$$
\begin{aligned}
G_{\widehat{\jmath}}\left(\tau_{1}, \tau_{1}^{\prime}\right)= & -\left\langle T_{\tau} \widehat{\jmath}\left(\tau_{1}\right) \widehat{\jmath}\left(\tau_{1}^{\prime}\right)\right\rangle \\
= & \left(\frac{e t}{N}\right)^{2} \sum_{\left\langle m_{1}, m_{2}\right\rangle} \sum_{\left\langle m_{1}^{\prime}, m_{2}^{\prime}\right\rangle}\left(m_{1}-m_{2}\right)\left(m_{1}^{\prime}-m_{2}^{\prime}\right) \\
& \times\left\langle T_{\tau} c_{m_{1}}^{\dagger}\left(\tau_{1}\right) c_{m_{2}}\left(\tau_{1}\right) c_{m_{1}^{\prime}}^{\dagger}\left(\tau_{1}^{\prime}\right) c_{m_{2}^{\prime}}\left(\tau_{1}^{\prime}\right)\right\rangle \\
= & \left(\frac{e t}{N}\right)^{2} \sum_{\left\langle m_{1}, m_{2}\right\rangle} \sum_{\left\langle m_{1}^{\prime}, m_{2}^{\prime}\right\rangle}\left(m_{1}-m_{2}\right)\left(m_{1}^{\prime}-m_{2}^{\prime}\right) \\
& \times\left.\left\langle T_{\tau} c_{m_{2}}\left(\tau_{2}\right) c_{m_{2}^{\prime}}\left(\tau_{2}^{\prime}\right) c_{m_{1}^{\prime}}^{\dagger}\left(\tau_{1}^{\prime}\right) c_{m_{1}}^{\dagger}\left(\tau_{1}\right)\right\rangle\right|_{\tau_{2}=\tau_{1}-, \tau_{2}^{\prime}=\tau_{1}^{\prime}-},
\end{aligned}
$$

where $m_{i}, m_{i}^{\prime}$ are integers labeling the sites of the 1D lattice. The two-particle Green function

$$
G\left(2,2^{\prime} ; 1,1^{\prime}\right)=\left\langle T_{\tau} c_{m_{2}}\left(\tau_{2}\right) c_{m_{2}^{\prime}}\left(\tau_{2}^{\prime}\right) c_{m_{1}^{\prime}}^{\dagger}\left(\tau_{1}^{\prime}\right) c_{m_{1}}^{\dagger}\left(\tau_{1}\right)\right\rangle
$$

for mutually independent fermions can be written in terms of one-particle Green functions [30]. Assuming $\langle\widehat{\jmath}\rangle=0$ in the absence of an electric field, the part of the two-particle Green function relevant for the evaluation of equation (11) reads [30]

$$
\begin{aligned}
G\left(2,2^{\prime} ; 1,1^{\prime}\right) & =-G\left(2,1^{\prime}\right) G\left(2^{\prime}, 1\right) \\
& =-\left\langle T_{\tau} c_{m_{2}}\left(\tau_{2}\right) c_{m_{1}^{\prime}}^{\dagger}\left(\tau_{1}^{\prime}\right)\right\rangle\left\langle T_{\tau} c_{m_{2}^{\prime}}\left(\tau_{2}^{\prime}\right) c_{m_{1}}^{\dagger}\left(\tau_{1}\right)\right\rangle .
\end{aligned}
$$

We use the Fourier transformation of the one-particle Green functions

$$
G\left(2,1^{\prime}\right)=\frac{1}{N} \sum_{k} \mathrm{e}^{\mathrm{i} k\left(m_{2}-m_{1}^{\prime}\right)} \frac{1}{\beta} \sum_{\omega_{\nu}} \mathrm{e}^{-\mathrm{i} \omega_{\nu}\left(\tau_{2}-\tau_{1}^{\prime}\right)} G\left(k, \mathrm{i} \omega_{\nu}\right)
$$

with $\omega_{\nu}=(2 \nu+1) \pi / \beta, k=2 \pi m / N$ (likewise for $G\left(2^{\prime}, 1\right)$ ), and the spectral representation

$$
G\left(k, \mathrm{i} \omega_{\nu}\right)=\int_{-\infty}^{\infty} \mathrm{d} \omega^{\prime} \frac{A\left(k, \omega^{\prime}\right)}{\mathrm{i} \omega_{\nu}-\omega^{\prime}},
$$

to obtain

$$
G_{\widehat{\jmath}}\left(\mathrm{i} \omega_{n}\right)=\int_{0}^{\beta} \mathrm{d}\left(\tau_{1}-\tau_{1}^{\prime}\right) \mathrm{e}^{\mathrm{i} \omega_{n}\left(\tau_{1}-\tau_{1}^{\prime}\right)} G_{\widehat{\jmath}}\left(\tau_{1}-\tau_{1}^{\prime}\right),
$$

where $\omega_{n}=2 n \pi / \beta$. Using equations (12)-(15) to express the rhs of equation (11), the Fourier transformation (16) is evaluated by carrying out the summations over $m_{i}, m_{i}^{\prime}$, and the integration and subsequent summation over the Matsubara frequencies $\omega_{\nu}$ [30]. We find

$G_{\widehat{\jmath}}\left(\mathrm{i} \omega_{n}\right)=\left(\frac{2 e t}{N}\right)^{2} \sum_{k}(\sin k)^{2} \int_{-\infty}^{\infty} \mathrm{d} \omega^{\prime} \int_{-\infty}^{\infty} \mathrm{d} \epsilon^{\prime} A\left(k, \omega^{\prime}\right) A\left(k, \epsilon^{\prime}\right) \frac{f\left(\omega^{\prime}\right)-f\left(\epsilon^{\prime}\right)}{\omega^{\prime}-\epsilon^{\prime}+\mathrm{i} \omega_{n}}$.

Here $f(x)=[\exp (\beta x)+1]^{-1}$ is the Fermi function. Using Dirac's identity

$$
\frac{1}{\omega+\omega^{\prime}-\epsilon^{\prime}+\mathrm{i} 0^{+}}=\frac{\mathcal{P}}{\omega+\omega^{\prime}-\epsilon^{\prime}}-\mathrm{i} \pi \delta\left(\omega+\omega^{\prime}-\epsilon^{\prime}\right),
$$

the analytical continuation $\mathrm{i} \omega_{n} \mapsto \omega+\mathrm{i} \delta$ gives in the limit $\delta \rightarrow 0^{+}$

$$
\begin{aligned}
\operatorname{Im} G_{\widehat{\jmath}}^{\mathrm{R}}(\omega)=- & \left(\frac{2 e t}{N}\right)^{2} \sum_{k}(\sin k)^{2} \int_{-\infty}^{\infty} \mathrm{d} \omega^{\prime} \int_{-\infty}^{\infty} \mathrm{d} \epsilon^{\prime} A\left(k, \omega^{\prime}\right) A\left(k, \epsilon^{\prime}\right) \\
& \times\left[f\left(\omega^{\prime}\right)-f\left(\epsilon^{\prime}\right)\right] \delta\left(\omega+\omega^{\prime}-\epsilon^{\prime}\right) .
\end{aligned}
$$


In particular, for $\omega>0$ and $T \rightarrow 0$, and introducing the Heaviside step function $\theta(x)$,

$\operatorname{Im} G_{\widehat{\jmath}}^{\mathrm{R}}(\omega)=-\pi\left(\frac{2 e t}{N}\right)^{2} \sum_{k}(\sin k)^{2} \int_{-\infty}^{0} \mathrm{~d} \omega^{\prime} A\left(k, \omega^{\prime}\right) A\left(k, \omega^{\prime}+\omega\right) \theta\left(\omega^{\prime}+\omega\right)$.

The real part of $\sigma(\omega)$ for $\omega>0$, given by

$\sigma^{\mathrm{reg}}(\omega)=\frac{4 \sigma_{0}}{\omega} \frac{1}{N} \sum_{k}(\sin k)^{2} \int_{-\infty}^{0} \mathrm{~d} \omega^{\prime} A\left(k, \omega^{\prime}\right) A\left(k, \omega+\omega^{\prime}\right) \theta\left(\omega+\omega^{\prime}\right)$,

is determined by the overlap of the electronic spectral functions for energies $\omega^{\prime}<0$ (relative to $\mu$ ) and $\omega^{\prime}+\omega>0$, respectively. Here we have defined $\sigma_{0}=a \pi(e t)^{2}$.

The neglect of vertex corrections in the current-current response function based on equation (13) leads to the factorisation into spectral functions in equation (21), which is equivalent to the exact $\mathrm{D}=\infty$ result commonly used in dynamical mean-field theory (DMFT) [31]. However, in contrast to the local approximation of DMFT, the spectral functions used here have a non-trivial momentum dependence [17].

\subsection{Limiting cases}

Weak coupling The explicit form of the WC electronic spectral function, calculated according to equations (A.1)-(A.4), was determined in [17]. Taking into account the condition $|\omega|<\omega_{0}\left(|\omega|>\omega_{0}\right)$ for the coherent (incoherent) part, and the explicit result (A.2) for the coherent part $A^{\mathrm{c}}(k, \omega)$, we find for the optical conductivity

$$
\begin{aligned}
\sigma^{\mathrm{reg}}(\omega)=\frac{4 \sigma_{0}}{\pi \omega} \int_{0}^{\pi} \mathrm{d} k(\sin k)^{2} \\
\times\left[z_{k} \theta\left(-\left(E_{k}-\mu\right)\right) \theta\left(\omega_{0}+E_{k}-\mu\right) \theta\left(E_{k}-\mu+\omega-\omega_{0}\right) A^{\mathrm{ic}}\left(k, E_{k}-\mu+\omega\right)\right. \\
\quad+z_{k} \theta\left(E_{k}-\mu\right) \theta\left(\omega_{0}-\left(E_{k}-\mu\right)\right) \theta\left(\omega-\left(E_{k}-\mu\right)-\omega_{0}\right) A^{\mathrm{ic}}\left(k, E_{k}-\mu-\omega\right) \\
\left.\quad+\int_{-\infty}^{0} \mathrm{~d} \omega^{\prime} A^{\mathrm{ic}}\left(k, \omega^{\prime}\right) A^{\mathrm{ic}}\left(k, \omega+\omega^{\prime}\right) \theta\left(-\omega^{\prime}-\omega_{0}\right) \theta\left(\omega+\omega^{\prime}-\omega_{0}\right)\right] .
\end{aligned}
$$

For the discussion of equation (22) in section 4, we have to recall [17] that the WC approximation of the incoherent one-electron spectral function $A^{\text {ic }}\left(k, \omega^{\prime}\right)$ is non-zero only for $\omega^{\prime} \in\left(-\omega_{0}-W-\mu,-\omega_{0}\right)$ or $\omega^{\prime} \in\left(\omega_{0}, \omega_{0}+W-\mu\right)$. The last term in equation (22), corresponding to transitions between these frequency intervals of the spectrum, is denoted in figure 1 as $D$.

Intermediate coupling Within the variational approach, the electronic spectral function is given by equation (A.5) with $g$ replaced by $\bar{\gamma} g$ and $\widetilde{W}=W \mathrm{e}^{-\bar{\gamma}^{2} g^{2}}$. The explicit expression for $\Sigma(k, \omega)$ determining the polaronic spectral function can be found in [17]. Using the definitions (A.6)-(A.9), we can write

$$
\int_{-\infty}^{0} \mathrm{~d} \omega^{\prime} \theta\left(\omega+\omega^{\prime}\right) A_{\mathrm{e}}\left(k, \omega^{\prime}\right) A_{\mathrm{e}}\left(k, \omega^{\prime}+\omega\right)=A+B+C+D
$$

In general all terms of equation (23) contribute to $\sigma^{\text {reg }}(\omega)$. We find

$$
\begin{aligned}
{\left[\sigma^{\mathrm{reg}}(\omega)\right]_{A}=\frac{4 \sigma_{0}}{\pi^{2} \omega} } & \mathrm{e}^{-2(\bar{\gamma} g)^{2}} \sum_{s \geq 1} \frac{(\bar{\gamma} g)^{2 s}}{s !} \int_{0}^{\pi} \mathrm{d} k(\sin k)^{2} z_{k} \\
& \times\left[\frac{z_{k_{1}}}{\left|\partial_{k_{1}} E_{k_{1}}\right|} \theta\left(E_{k}+\eta\right) \theta\left(\omega_{0}-\left(E_{k}+\eta\right)\right) \theta\left(-E_{k_{1}}-\eta\right) \theta\left(\omega_{0}+E_{k_{1}}+\eta\right)\right.
\end{aligned}
$$




$$
\begin{aligned}
& \left.+\frac{z_{k_{2}}}{\left|\partial_{k_{2}} E_{k_{2}}\right|} \theta\left(-E_{k}-\eta\right) \theta\left(\omega_{0}+E_{k}+\eta\right) \theta\left(E_{k_{2}}+\eta\right) \theta\left(\omega_{0}-\left(E_{k_{2}}+\eta\right)\right)\right] \\
& +\frac{2 \sigma_{0}}{\omega \pi^{2}} \mathrm{e}^{-2(\bar{\gamma} g)^{2}} \sum_{s \geq 1} \frac{2^{s}(\bar{\gamma} g)^{2 s}}{s !}\left(1-2^{1-s}\right) \int_{0}^{\pi} \mathrm{d} k z_{k} \frac{z_{k^{\prime}}}{\left|\partial_{k^{\prime}} E_{k^{\prime}}\right|} \\
& \times \theta\left(-E_{k}-\eta\right) \theta\left(\omega_{0}+E_{k}+\eta\right) \theta\left(E_{k^{\prime}}+\eta\right) \theta\left(\omega_{0}-\left(E_{k^{\prime}}+\eta\right)\right)
\end{aligned}
$$

where

$$
\begin{aligned}
{\left[\frac{z_{k}}{\left|\partial_{k} E_{k}\right|}\right]^{-1}=\mid \widetilde{W} } & \sin k\left\{1-\frac{2}{\pi} \cos k \sum_{s \geq 1} \frac{(\bar{\gamma} g)^{2 s}}{s !} \mathcal{P} \int_{-\widetilde{W}}^{\widetilde{W}} \frac{\mathrm{d} \xi}{\sqrt{1-(\xi / \widetilde{W})^{2}}}\right. \\
& \left.\times\left[\frac{\theta(\xi+\eta)}{E_{k}-s \omega_{0}-\xi}+\frac{\theta(-\xi-\eta)}{E_{k}+s \omega_{0}-\xi}\right]\right\}+\frac{2}{\pi} \bar{\gamma}(1-\bar{\gamma}) E_{\mathrm{P}} \sin k \\
& \times \mathcal{P} \int_{-\widetilde{W}}^{\widetilde{W}} \frac{\mathrm{d} \xi}{\sqrt{1-(\xi / \widetilde{W})^{2}}}\left[\frac{\theta(\xi+\eta)}{E_{k}-\omega_{0}-\xi}-\frac{\theta(-\xi-\eta)}{E_{k}+\omega_{0}-\xi}\right] \mid .
\end{aligned}
$$

Here $k_{1}, k_{2} \in(0, \pi)$ are determined by the conditions $E_{k_{1}}=E_{k}-\left(\omega-s \omega_{0}\right)$ and $E_{k_{2}}=E_{k}+\left(\omega-s \omega_{0}\right)$, whereas $k^{\prime}>k_{\mathrm{F}}$ is given as the solution of $E_{k^{\prime}}=E_{k}+\left(\omega-s \omega_{0}\right)$.

For the other contributions to equation (23), we have

$$
\begin{aligned}
& B=\mathrm{e}^{-(\bar{\gamma} g)^{2}} z_{k} A_{\mathrm{e}}^{\mathrm{ic}}(\left.k, \omega+E_{k}+\eta\right) \theta\left(\omega+E_{k}+\eta\right) \theta\left(-E_{k}-\eta\right) \theta\left(\omega_{0}+E_{k}+\eta\right) \\
&+ \mathrm{e}^{-(\bar{\gamma} g)^{2}} \sum_{s \geq 1} \frac{(\bar{\gamma} g)^{2 s}}{s !} \frac{1}{\pi} \int_{0}^{\pi} \mathrm{d} k^{\prime} z_{k^{\prime}} A_{\mathrm{e}}^{\mathrm{ic}}\left(k, \omega+E_{k^{\prime}}+\eta-s \omega_{0}\right) \\
& \times \theta\left(\omega+E_{k^{\prime}}+\eta-s \omega_{0}\right) \theta\left(-E_{k^{\prime}}-\eta\right) \theta\left(E_{k^{\prime}}+\eta+\omega_{0}\right), \\
& C=\mathrm{e}^{-(\bar{\gamma} g)^{2}} z_{k} A_{\mathrm{e}}^{\mathrm{ic}}\left(k, E_{k}+\eta-\omega\right) \theta\left(\omega-E_{k}-\eta\right) \theta\left(E_{k}+\eta\right) \theta\left(\omega_{0}-E_{k}-\eta\right) \\
&+\mathrm{e}^{-(\bar{\gamma} g)^{2}} \sum_{s \geq 1} \frac{(\bar{\gamma} g)^{2 s}}{s !} \frac{1}{\pi} \int_{0}^{\pi} \mathrm{d} k^{\prime} z_{k^{\prime}} A_{\mathrm{e}}^{\mathrm{ic}}\left(k, E_{k^{\prime}}+\eta+s \omega_{0}-\omega\right) \\
& \times \theta\left(\omega-E_{k^{\prime}}-\eta-s \omega_{0}\right) \theta\left(E_{k^{\prime}}+\eta\right) \theta\left(\omega_{0}-E_{k^{\prime}}-\eta\right),
\end{aligned}
$$

and

$$
D=\int_{-\infty}^{0} \mathrm{~d} \omega^{\prime} A_{\mathrm{e}}^{\mathrm{ic}}\left(k, \omega^{\prime}\right) A_{\mathrm{e}}^{\mathrm{ic}}\left(k, \omega+\omega^{\prime}\right) \theta\left(\omega+\omega^{\prime}\right)
$$

so that

$$
\left[\sigma^{\mathrm{reg}}(\omega)\right]_{B+C+D}=\frac{4 \sigma_{0}}{\pi \omega} \int_{0}^{\pi} \mathrm{d} k(\sin k)^{2}(B+C+D) .
$$

The parts of the electronic spectral function denoted by $A_{\mathrm{e}}^{\mathrm{ic}}\left(k, \omega^{\prime}\right)$, defined in equation (A.8), are determined by the incoherent parts of the polaronic spectral function.

Strong coupling For $g^{2} \gg 1$, small polarons are the correct fermionic quasiparticles, and the calculations corresponding to equations (A.1)-(A.4) are done using the small-polaron Hamiltonian defined by the coefficients (3) (see [17] for explicit results). In this regime, the zeroth-order small-polaron approximation-with the spectrum consisting only of coherent states in the polaron band-is applicable and the polaronic spectral weights $z_{k} \approx 1$ [17]. Consequently, it is sufficient to keep only the term A, and SC expressions corresponding to equations (24) and (25) can be obtained by setting $\bar{\gamma}=1$. 
(I)

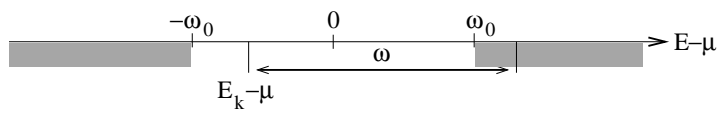

(II)

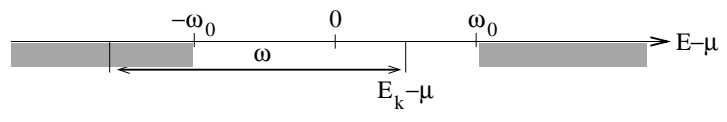

(D)

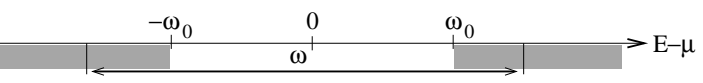

Figure 1. Illustration of the transitions contributing to equation (22).

(A1)

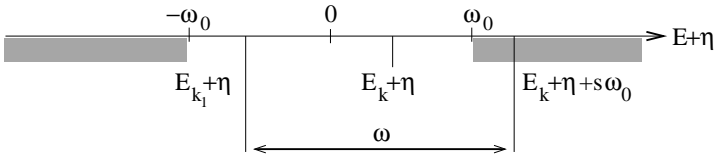

(A2)

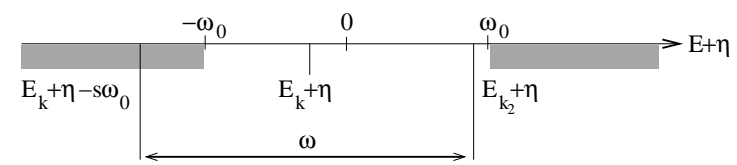

(A3)

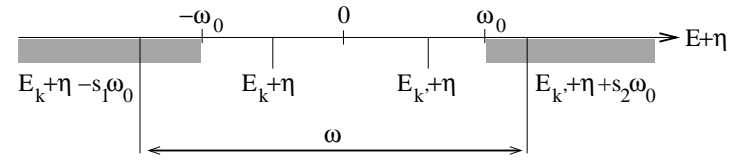

(B)

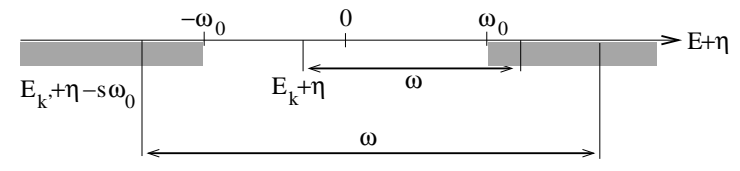

(C)

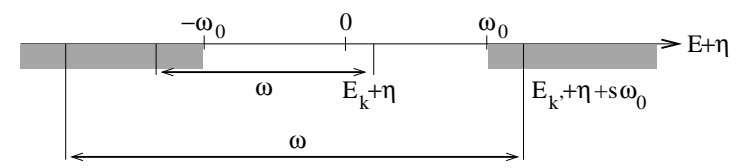

Figure 2. Transitions contributing to equations (24), (26) and (27). Equation (28) corresponds to figure 1 (D) with $-\mu$ replaced by $\eta$.

The rather complicated explicit formulas for the optical conductivity presented above are visualised in figures 1 and 2 for the $\mathrm{WC}$ and the SC/IC cases, respectively. In particular, these pictures show that non-zero values of $\sigma^{\mathrm{reg}}(\omega)$ are restricted to $\omega>\omega_{0}$. The existence of such a threshold for the optical conductivity at $T=0$ was already recognised in [20] on the basis of WC calculations in the framework of the continuous polaron model.

\section{Results}

We now come to a discussion of numerical results obtained from the analytical expressions derived in section 3, which will be compared to exact data for one electron. It is instructive to first consider the kinetic energy and the Drude weight, related to $\sigma^{\text {reg }}(\omega)$ by the f-sum rule. 


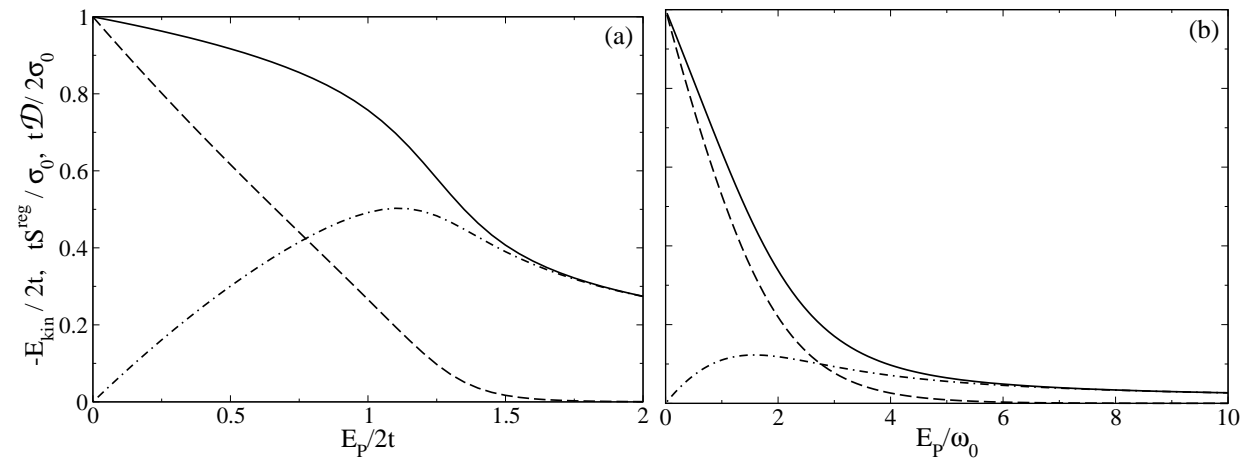

Figure 3. Numerical results for the kinetic energy $E_{\text {kin }}(-)$, the Drude weight $\mathcal{D}(---)$, and the integrated spectral weight $S^{\mathrm{reg}}(-\cdot-$, equation (33)), for one electron as a function of electron-phonon coupling strength. Here (a) $\omega_{0} / t=0.4$ and (b) $\omega_{0} / t=4$.

\subsection{Integral quantities}

In a tight-binding system with the kinetic energy operator

$$
\widehat{T}=-t \sum_{\langle i, j\rangle} c_{i}^{\dagger} c_{j}
$$

and the current density operator (7), it follows that $[32,33]$

$$
-e^{2} a \varepsilon_{\text {kin }}=\frac{1}{\pi} \int_{-\infty}^{\infty} \mathrm{d} \omega \sigma(\omega)=\frac{2}{\pi} \int_{0}^{\infty} \mathrm{d} \omega \operatorname{Re} \sigma(\omega),
$$

by symmetry of $\sigma(\omega)$. Here, $\varepsilon_{\text {kin }}=\langle\widehat{T}\rangle / N$ represents the kinetic energy per lattice site.

For $\omega>0$ and $T=0$, the electron-phonon interaction implied in the Holstein model considered here gives a non-zero $\operatorname{Re} \sigma(\omega)$ only if $\omega>\omega_{0}$. At $\omega=0, \operatorname{Re} \sigma(\omega)$ has a singularity to be deduced in Appendix B. Writing

$$
\operatorname{Re} \sigma(\omega)=\mathcal{D} \delta(\omega)+\sigma^{\mathrm{reg}}(\omega),
$$

where $\mathcal{D}$ is the so-called Drude weight, and defining $S^{\text {reg }} \equiv S^{\mathrm{reg}}(\infty)$ with

$$
S^{\mathrm{reg}}(\omega)=\int_{0}^{\omega} \mathrm{d} \omega^{\prime} \sigma^{\mathrm{reg}}\left(\omega^{\prime}\right)
$$

the f-sum rule (31) takes the form

$$
-e^{2} a \varepsilon_{\mathrm{kin}}=\frac{1}{\pi} \mathcal{D}+\frac{2}{\pi} S^{\mathrm{reg}}
$$

The kinetic energy may be determined independent of the rhs of equation (34) from the electronic spectral function as [34]

$$
\varepsilon_{\text {kin }}=\frac{1}{N} \sum_{k} \int_{-\infty}^{0} \mathrm{~d} \omega \varepsilon_{k} A(k, \omega), \quad \varepsilon_{k}=-2 t \cos k .
$$

In the case of one electron in a lattice with $N$ sites (i.e., with carrier concentration $n=1 / N), \varepsilon_{\text {kin }}$ is equal to the kinetic energy $E_{\text {kin }}$ of one electron divided by $N$. To obtain non-zero results, both sides of equation (34) are multiplied by $N$ before taking the limit $N \rightarrow \infty$. Accordingly, using the spectral representation at $T=0$, we have for $\omega>0$

$$
\sigma^{\mathrm{reg}}(\omega)=\frac{a \pi}{\omega} \sum_{E_{m}>E_{0}}\left|\left\langle\psi_{m}|\widehat{\jmath}| \psi_{0}\right\rangle\right|^{2} \delta\left[\omega-\left(E_{m}-E_{0}\right)\right]
$$


Optical conductivity of polaronic charge carriers

Table 1. Analytical results for the kinetic energy $-\varepsilon_{\text {kin }} / t$ for $\omega_{0} / t=0.4$, QMC data for $N=16, \beta t=10, \Delta \tau=0.05$ [7], and ED data for $N=10\left({ }^{*}\right.$-not fully converged with respect to the number of phonons $M=25$ ) [16].

\begin{tabular}{c|c|c|c}
\hline Parameters & Analytical & QMC & ED \\
\hline$E_{\mathrm{P}} / t=0.1, n=0.1$ & 0.202 (WC) 0.211 (IC) & 0.190 & 0.1986 \\
$E_{\mathrm{P}} / t=0.1, n=0.3$ & 0.509 (WC) 0.518 (IC) & 0.504 & 0.5208 \\
$E_{\mathrm{P}} / t=0.1, n=0.4$ & 0.600 (WC), 0.609 (IC) & 0.596 & 0.5824 \\
\hline$E_{\mathrm{P}} / t=2.0, n=0.1$ & 0.155 (IC) & $0.136 \pm 0.002$ & 0.1517 \\
$E_{\mathrm{P}} / t=2.0, n=0.3$ & 0.382 (IC) & $0.36 \pm 0.01$ & 0.4070 \\
$E_{\mathrm{P}} / t=2.0, n=0.4$ & 0.397 (IC) & $0.43 \pm 0.02$ & 0.4531 \\
\hline$E_{\mathrm{P}} / t=4.0, n=0.4$ & 0.13 (SC) & $0.19 \pm 0.01$ & $0.2318^{*}$ \\
\hline
\end{tabular}

with the current operator $\widehat{\jmath}=-\mathrm{i} e t \sum_{i}\left(c_{i}^{\dagger} c_{i+1}-c_{i+1}^{\dagger} c_{i}\right)$ for a single electron. Here $\left|\psi_{m}\right\rangle$ denotes the $m$-th excited state with energy $E_{m}$. In comparison to equations (7), (8) a factor $1 / N$ is omitted in equation (36).

According to Kohn's formula, the Drude weight of the Holstein model with one electron can be calculated from the effective mass $m^{*}$ as ( $E_{k}$ is the polaron band dispersion) [35]

$$
\mathcal{D}=\left.e^{2} \pi a \partial_{k}^{2} E_{k}\right|_{k=0}=e^{2} \pi a \frac{1}{m^{*}} .
$$

The evaluation of $\mathcal{D}$ via the $\mathrm{f}$-sum rule, and the determination of $m^{*}$ are independent computations, so that equation (37) may be used to validate the numerics.

4.1.1. One electron We adopt the basis construction of [36], which allows for the calculation of results that are variational in the thermodynamic limit, and find that the f-sum rule is fulfilled to at least six digits for the parameters shown. For spectral properties a Chebyshev expansion method is used [37].

The dependence of $E_{\text {kin }}, S^{\text {reg }}$ and $\mathcal{D}$ on the electron-phonon coupling strength reflects the well-known crossover from a large polaron at weak coupling to a small polaron at strong coupling [38]. As expected, the results in figure 3 reveal a significant dependence on the adiabaticity ratio $\omega_{0} / t$. The adiabatic regime (figure 3(a)) is characterised by rather pronounced decrease of $E_{\mathrm{kin}}$ and $\mathcal{D}$ (increase of $m^{*}$ ) in the vicinity of the point $\lambda=1$ near which the crossover occurs, which is compensated by an increase of $S^{\text {reg }}$ due to enhanced incoherent scattering. In contrast, in the non-adiabatic regime (figure 3(b)), these changes occur over a much larger range of the relevant coupling constant $g^{2}$.

4.1.2. Many electrons Equation (35) turns out to be numerically problematic in the SC regime, and $\varepsilon_{\text {kin }}$ is instead calculated from equation (B.17). An approximation for $\mathcal{D}$ is given by equation (B.10).

To test the reliability of the spectral functions obtained from the analytical approach we compare in table 1 the analytical kinetic energy $\varepsilon_{\text {kin }}$ for different parameter sets to numerical data from quantum Monte Carlo (QMC) [7] and exact diagonalisation (ED) [16]. We restrict ourselves to the adiabatic regime since the analytical approach generally works better for $\omega_{0} / t \gg 1$ [17]. The agreement is satisfactory in all cases, with the deviations of the analytical results for $E_{\mathrm{P}} / t=2$ originating to some degree in the missing spectral weight in $A(k, \omega)$ (see below). The SC approach slightly underestimates the kinetic energy for $E_{\mathrm{P}} / t=4$, a coupling which does not fall into the true SC regime (see also figure 5(c)). 


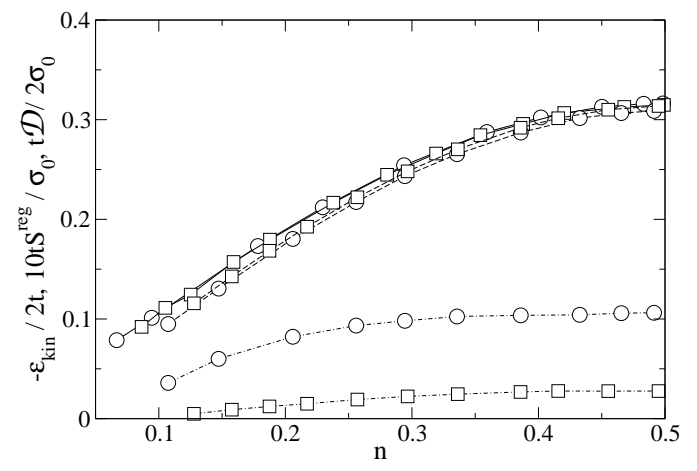

Figure 4. Analytical results for the kinetic energy $\varepsilon_{\text {kin }}(-)$, the Drude weight $\mathcal{D}(---)$, and the integrated spectral weight $S^{\text {reg }}(-\cdot-)$ as a function of band filling $n$. Here $E_{\mathrm{P}} / t=0.1$ and $\omega_{0} / t=0.4(\mathrm{O})$ respectively $\omega_{0} / t=4(\square)$.

Figure 4 shows the dependence of $\varepsilon_{\text {kin }}, \mathcal{D}$, and $S^{\text {reg }}$ on carrier density for $E_{\mathrm{P}} / t=0.1$. Whereas the kinetic energy and the Drude weight are almost identical in the adiabatic and non-adiabatic regime, the regular part $S^{\text {reg }}$ is substantially larger for $\omega_{0} / t<1$ owing to enhanced incoherent scattering of carriers by phonons, as is also observed in the one-electron case (figure 3(a)). The weak coupling leads to very small $S^{\text {reg }}$ (note the different scale used for $S^{\text {reg }}$ ) and hence requires-via the f-sum rule-that $-\varepsilon_{\text {kin }} / t \approx t \mathcal{D} / \sigma_{0}$. A comparison of $S^{\text {reg }}$ to exact IC results [16] reveals similar deviations as for $\varepsilon_{\text {kin }}$.

The approximations for the Drude weight $\mathcal{D}$ derived in Appendix B yield reliable results in the $\mathrm{WC}$ and $\mathrm{SC}$ cases, whereas the dependence of $\mathcal{D}$ on $E_{\mathrm{P}}$ for intermediate coupling is not properly described. Alternatively, an estimate for $\mathcal{D}$ may be obtained from the sum rule, but will be affected by the deviations of $\varepsilon_{\text {kin }}$ and $S^{\text {reg }}$ from exact results.

\subsection{Optical response}

4.2.1. One electron Although the optical conductivity for one electron has been discussed before [38], we shall begin with this case as it provides a framework for the finite-density case, and another test for the analytical theory. We focus on the adiabatic regime $\omega_{0} / t<1$ relevant for many polaronic materials.

Figure 5 shows numerical and analytical results for $\sigma^{\text {reg }}(\omega)$ for $\omega_{0} / t=0.4$ and three different values of $E_{\mathrm{P}}$. In order to compare with the one-electron case, we have chosen $n=0.1$ in the analytical approach, and scaled the results to yield the exact one-electron value for $S^{\text {reg. }}$. The dependence of $\sigma^{\text {reg }}(\omega)$ and $S^{\text {reg }}$ on $n$ will be discussed below.

As anticipated from the analytical results (cf equations (22) and (24)), there exists a minimal absorption energy (absorption threshold) $\omega=\omega_{0}$. Another striking feature is the change from an asymmetric spectrum in the large polaron regime to a (more) symmetric spectrum in the small-polaron regime also observed experimentally [5].

Starting with weak coupling $E_{\mathrm{P}}=0.1$ in figure 5(a), we find a pronounced absorption signal near $\omega=\omega_{0}$, followed by a continuous decrease. The analytical results from the IC approximation reproduce the main features (notice the good agreement for $S^{\text {reg }}(\omega)$ ), with the sharp low-energy peaks smeared out. As illustrated by the WC results in the inset (in good agreement with the IC results), this smearing depends on the number of $k$-values used in the integration ( $N=31$ and 801 , respectively). The inset also contains analytical results for $\mu \rightarrow E_{0}^{+}$, corresponding to the one-electron case [18], for which a non-zero WC result is 

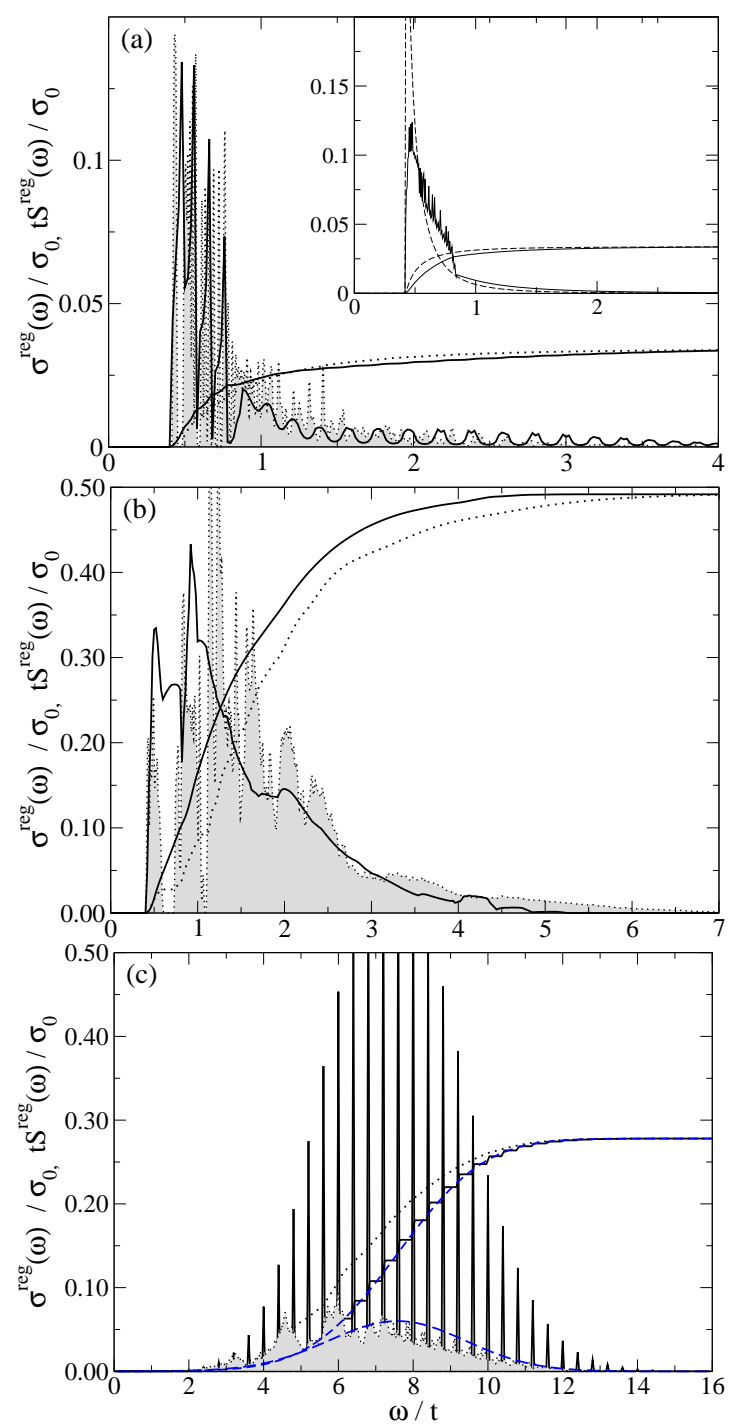

Figure 5. Numerical one-electron results $(\ldots \ldots)$ for the regular part of the optical conductivity $\sigma^{\mathrm{reg}}(\omega)$ and the integrated spectral weight $S^{\mathrm{reg}}(\omega)$, and analytical results ( - , scaled to give the same $S^{\mathrm{reg}}$ ) for $n=0.1$ from the IC approximation with (a) $R=2.4$, (b) $R=2.1$, (c) $R=0.1$. Here $\omega_{0} / t=0.4$ and (a) $E_{\mathrm{P}} / t=0.1$, (b) $E_{\mathrm{P}} / t=2$, and (c) $E_{\mathrm{P}} / t=4$. The inset in (a) compares the WC analytical result for $n=0.1$ with that obtained for $n \rightarrow 0$ (- - -, see text), both rescaled to $S^{\text {reg }}$ for one electron. Panel (c) includes the small-polaron result of [39] (- - - ).

obtained by using the zero-density spectral function in equation (22). Obviously, small peaks in $\sigma^{\text {reg }}(\omega)$ are further washed out in this limit. The sharp peaks in the exact numerical results are due to the use of a finite number of poles in equation (36).

Turning to the IC results ( $E_{\mathrm{P}} / t=2$, close to the critical coupling for the small-polaron crossover) depicted in figure 5(b), we find low-energy peaks split off from the high-energy part and separated by $\omega_{0}$. Besides, the long high-energy tail of both the exact and analytical curves is reminiscent of experimental data on manganites [5] and also $\mathrm{TiO}_{2}$ [40], and its 


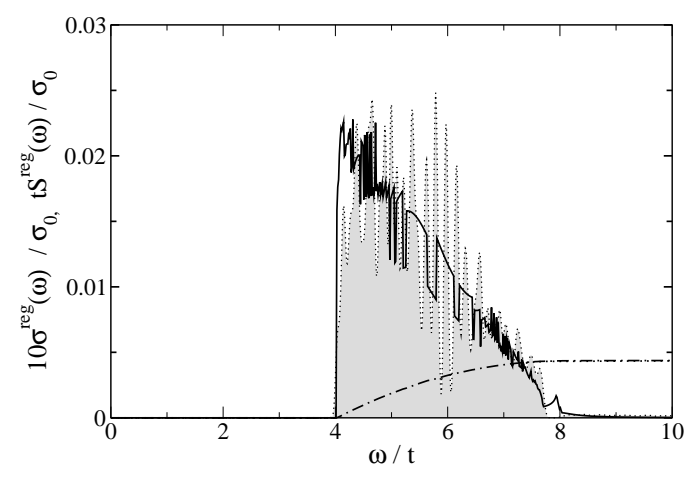

Figure 6. Numerical results for $\sigma^{\mathrm{reg}}(\omega)$ for one electron $(\cdots \cdots)$, and analytical results $\left(-, n=0.1\right.$, scaled to the same $\left.S^{\mathrm{reg}}\right)$ from the WC approximation. Here $\omega_{0} / t=4$ and $E_{\mathrm{P}} / t=0.1$. Also shown is $S^{\mathrm{reg}}(\omega)$.

occurrence in the IC regime points toward the inadequacy of standard small-polaron theory for such materials.

Finally, for $E_{\mathrm{P}} / t=4$ (figure 5(c)), we find a maximum close to that of the smallpolaron result [39] which peaks at $2 E_{\mathrm{P}}$ and is included for reference, with the parameter $\bar{\sigma}_{0}$ chosen so as to get the same $S^{\mathrm{reg}}$. For technical reasons, the optical conductivity in the SC regime (given analytically by a sum of $\delta$-peaks) is calculated using an artificial broadening (see many-electron case).

The exact numerical results, however, reveal that the parameters chosen do not fall into the true small-polaron regime, as indicated by a slight asymmetry, a maximum clearly below $2 E_{\mathrm{P}}$, and three low-energy peaks (not related to finite-size effects) which diminish with increasing $E_{\mathrm{P}}$. The latter originate from transitions between the (dispersive) coherent band and dispersionless phonon satellites (cf figure 9(b) of [17]), and are absent in the analytical results probably due to an overestimation of band-narrowing in the adiabatic regime. The possibility of such overestimation by the Lang-Firsov small-polaron theory in the adiabatic case was qualitatively illustrated for the two-site model in [41], and the analytical results of figure 5(c) are expected in the SC (small-polaron) regime.

Figure 6 shows $\sigma^{\text {reg }}(\omega)$ at weak coupling in the non-adiabatic regime, taking $\omega_{0} / t=4$. As multi-phonon excitations have small weight due to $g^{2} \ll 1$, the absorption is virtually restricted to the interval $\left[\omega_{0}, 2 \omega_{0}\right]$. Here the analytical WC approach-restricted to onephonon excitations, see equation (22) - yields even better agreement than in figure 5(a).

Overall, the analytical approach is capable of reproducing the main features of the optical conductivity in the low-density regime, including the increase of the frequency range for absorption (figure 5). Furthermore, in accordance with figure 3, $S^{\text {reg }}$ is small at weak coupling, takes on a maximum in the IC regime, and decreases again due to the suppression of $\varepsilon_{\text {kin }}$ (via the sum rule) at strong coupling.

4.2.2. Many electrons There are some issues concerning the analytical results shown here which deserve attention. First, we pointed out in [17] that the total spectral weight $w=\int \mathrm{d} k \int \mathrm{d} \omega A(k, \omega) \leq 1$ contained in the spectral function (i.e., its norm) provides a first indication for the quality of the analytical approximation for a given set of parameters.

The IC approach reproduces the WC (SC) approximation in the WC (SC) regime [17]. In the vicinity of these limits, it yields results very similar to the WC/SC approximation, with $w \lesssim 1$. In the true IC regime $\left(\lambda=E_{\mathrm{P}} / 2 t \approx 1\right.$ or $\left.g^{2}=E_{\mathrm{P}} / \omega_{0} \approx 1\right)$, the norm $w$ becomes 

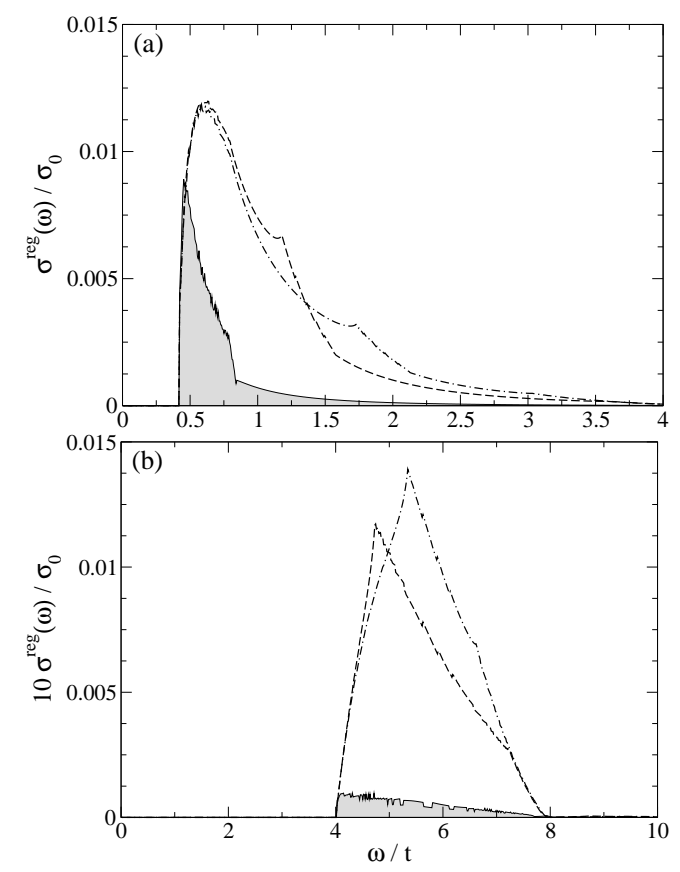

Figure 7. Analytical results for $\sigma^{\mathrm{reg}}(\omega)$ from the WC approximation for $n=0.1$ ( - ), $n=0.3(--)$ and $n=0.4(-\cdot-)$. Here $E_{\mathrm{P}} / t=0.1$, (a) $\omega_{0} / t=0.4$ and (b) $\omega_{0} / t=4$. The corresponding results for $S^{\text {reg }}$ are given in figure 4 .

as small as 0.7 in the worst case considered (for the parameters of figure 11(a)), with $w$ also depending on the radius $R$, whose "optimal" value is determined by minimising the energy within the Hartree approach. Despite these deviations, observables such as the kinetic energy (see table 1) or the total energy (see figure 13(a) in [17]) are in satisfactory agreement with exact results. Moreover, the optical conductivity - calculated from the spectral functionis mainly determined by states near the Fermi level, so that missing high-energy incoherent processes are irrelevant, and $w<1$ does not necessarily lead to poor results.

For some parameters, the spectral functions obtained from the IC approach exhibits small regions in the $(k, \omega)$ plane where $A(k, \omega)<0$ [17]. To calculate the optical conductivity, we have replaced such values by zero.

Finally, in the SC regime, an artificial "softening" proportional to the energy resolution is imposed on the $\theta$ functions in equation (24) which affects the integrated weight $S^{\text {reg }}$. Nevertheless, a consistent value for $S^{\text {reg }}$, and hence the normalisation of $\sigma^{\text {reg }}(\omega)$, can be obtained using equation (B.16).

Weak coupling We begin with the density dependence of $\sigma^{\text {reg }}(\omega)$. Figure 7 shows results for $E_{\mathrm{P}} / t=0.1$, and we first consider the adiabatic case $\omega_{0} / t=0.4$ in panel (a). The spectra exhibit several non-trivial features. Apart from the absorption threshold at $\omega_{0}$, we find an asymmetric peak exhibiting small but noticeable wiggles which extend over an interval $\omega_{0}$ centred at the maximum, and diminish with increasing $n$.

The origin of this feature are transitions of type II, as can be seen by separating the contributions I and II to equation (22), and looking at the corresponding spectral function $A(k, \omega)$ shown in figure 8. Non-zero contributions to the second term in equation (22) for 


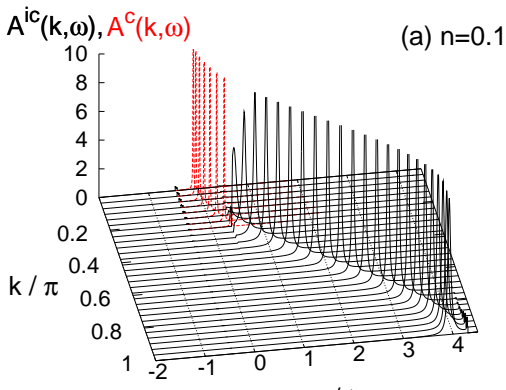

$\omega / \mathrm{t}$

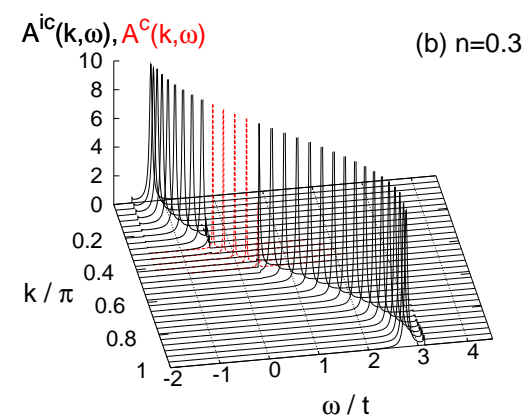

$\omega / \mathrm{t}$

Figure 8. (colour online) Coherent $\left(A^{\mathrm{c}},---\right)$ and incoherent $\left(A^{\mathrm{ic}},-\right)$ parts of the WC electronic spectral function for $\omega_{0} / t=0.4$ and $E_{\mathrm{P}} / t=0.1$ [17].

fixed $\omega$ correspond to the range of $k$ for which the coherent states with energy $E_{k}$ above the Fermi level may be connected to $A^{\text {ic }}\left(k, \omega^{\prime}\right)$ with $\omega^{\prime} \in\left(-\omega_{0}-W-\mu,-\omega_{0}\right)$. The absorption starts at $\omega_{\min }=\omega_{0}$ corresponding to $k_{\min }=k_{\mathrm{F}}$, whereas the upper cut-off $\omega_{\max }=2 \omega_{0}+|-W+\mu|$ for the adiabatic case (figure 7(a)) corresponds to $k_{\max }$ determined by $E_{k_{\max }}-\mu=\omega_{0}$. In the anti-adiabatic case, for which the entire coherent band lies inside the interval $\left(-\omega_{0}, \omega_{0}\right), \omega_{\max }=\omega_{0}+2 W$ corresponds to the highest energy $W-\mu$ with $k_{\text {max }}=\pi$.

The shape of the absorption curve in the interval $\left(\omega_{\min }, \omega_{\max }\right)$ depends on the $k$-interval contributing to the transition II for fixed $\omega$, and the corresponding values of $A^{\text {ic }}\left(k, E_{k}-\mu-\omega\right)$. For the anti-adiabatic case, figure 7(b) shows sharp maxima at the $n$-dependent positions $\omega=\omega_{0}+|-W-\mu|$, determined by $k_{\min }=k_{\mathrm{F}}$ and $k_{\max }$ fulfilling $E_{k_{\max }}-\mu=|-W-\mu|$.

For $n=0.1$, there is very little weight contained in incoherent states below $\mu$ (figure 8(a)). In fact, there exists only a single, small and narrow peak with negligible dispersion, and transitions from the latter to a coherent state can occur for $\omega_{0} \leq \omega \leq 2 \omega_{0}$, i.e., for final states between $E_{k}-\mu=0$ and the upper edge of the coherent band $E_{k}-\mu=\omega_{0}$. The wiggles, more pronounced for smaller numbers of $k$-values, result from transitions to the discrete $\delta$-peaks of the coherent spectrum.

Above $\omega=2 \omega_{0}$, for $n=0.1$, the transitions of type II fall off quickly with increasing $\omega$ and go to zero for $\omega>2 \omega_{0}+|-W-\mu| \approx 0.87$. In contrast, for larger $n$, the energy interval of incoherent states below the Fermi level $|-W-\mu|$ (and the weight contained in the latter) is significantly larger, yielding the cut-offs of $\omega \approx 1.6$ and $\omega \approx 2.15$. The high-frequency optical response is much smoother since the incoherent parts of the spectral function are broadened proportional to $E_{\mathrm{P}}$, and $S^{\mathrm{reg}}(\omega)$ increases in accordance with figure 4 .

Finally, the long high-energy tail in $\sigma^{\mathrm{reg}}(\omega)$ seen in figure 7(a) for all $n$ is due to incoherent excitations of type $D$ (figure 1 ), which have a maximum energy of $2\left(W+\omega_{0}\right)$ (within the WC approximation).

Strong coupling Figure 2 illustrates the contributions to $\sigma^{\mathrm{reg}}(\omega)$ in the SC regime, where the polaron band lies entirely in the interval $\left(-\omega_{0}, \omega_{0}\right)$. Equations (A1)-(A3) represent transitions between the states in the polaron band accompanied by multi-phonon processes. The processes (B) and (C), corresponding to transitions between the incoherent polaron spectrum and the polaron band states, again involving multiple phonons, are expected to become important for intermediate coupling.

Examining the analytical expressions, the most important processes in the true SC limit 


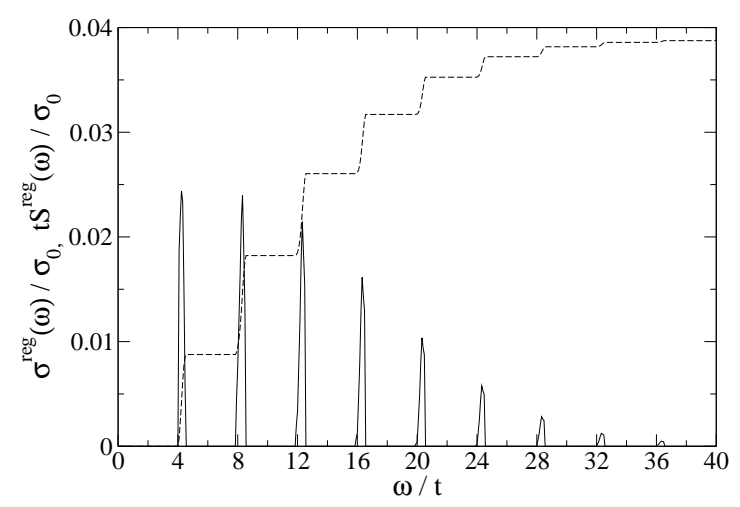

Figure 9. Analytical results for $\sigma^{\mathrm{reg}}(\omega)(-)$ and $S^{\mathrm{reg}}(\omega)$ (- - -) from the SC approximation. Here $\omega_{0} / t=4, E_{\mathrm{P}} / t=8$ and $n=0.4$.

$(\lambda, g \gg 1)$ appear to be those of type (A3), with the dominating (with respect to $\mathrm{e}^{-g^{2}}$ ) contribution to $\sigma^{\text {reg }}(\omega)$ given by equation (B.14). In this case, $\sigma^{\text {reg }}(\omega)$ consists of sharp peaks at $\omega=s \omega_{0}$ with heights modulated by the Poisson distribution with parameter $2 g^{2}$, as may be seen in figure 5(c). The maximum of the conductivity lies near the maximum of the Poisson distribution determined by $s=2 g^{2}=2 E_{\mathrm{P}} / \omega_{0}$.

The peak heights in the anti-adiabatic SC case $\left(g^{2}=2\right)$ shown in figure 9 can be understood by evaluating the relative weights in front of the integral on the rhs of equation (B.14), yielding $1,1,8 / 9$ for $s=1,2,3$, respectively.

The picture of the optical conductivity implied by our findings agrees with results obtained previously in the framework of small-polaron theory at $T=0[13,42]$. The separated peaks may be smeared out by means of an additional "smoothing mechanism" [42] or due to limited experimental resolution. The evolution of the small-polaron absorption spectrum towards a smooth function in dependence on the local-oscillator damping and temperature was considered in [43], and Emin [39] has presented the absorption spectrum in the form of a Gaussian centered at $2 E_{\mathrm{P}}$, assuming the broadening of the energy levels of the localised polaron states.

Crossover from weak to strong coupling Figure 10 illustrates the crossover from weak to intermediate to strong coupling for a fixed density $n=0.4$ in the adiabatic regime $\omega_{0} / t=0.4$ within the IC/SC approach. The corresponding spectral functions can be found in figure 14 of [17], and we use the same values of the parameter $R$ which decreases with increasing $E_{\mathrm{P}}$ reflecting a decrease of the polaron size.

The position of the maximum in $\sigma^{\text {reg }}(\omega)$ changes significantly as a function of $E_{\mathrm{P}}$. For weak coupling, it is located just above the absorption threshold at $\omega=\omega_{0}$ (figure 10(a)), whereas for $E_{\mathrm{P}} / t=2$ it lies near $1.5 \omega_{0}$. Nevertheless, the frequency range for absorption in panels (a) and (b) is very similar. For $E_{\mathrm{P}} / t=4$, similar to the low-density case shown in figure $5(\mathrm{c}), \sigma^{\mathrm{reg}}(\omega)$ peaks near the small-polaron value $2 E_{\mathrm{P}}$. The analytical approach overestimates the band narrowing, yielding SC behaviour even for a value $E_{\mathrm{P}} / t=4$ lying at the border to the SC region. Since for this value of $E_{\mathrm{P}}$ the IC and $\mathrm{SC}$ approximations yield virtually identical results, we have used the $\mathrm{SC}$ approximation to obtain a consistent value of $S^{\mathrm{reg}}$. The similarity of the SC results for $n=0.1$ in figure 5(c) and $n=0.4$ in figure 10 (c) is due to the rather weak density dependence of our analytical results pointed out before in the case of the spectral function [17]. 

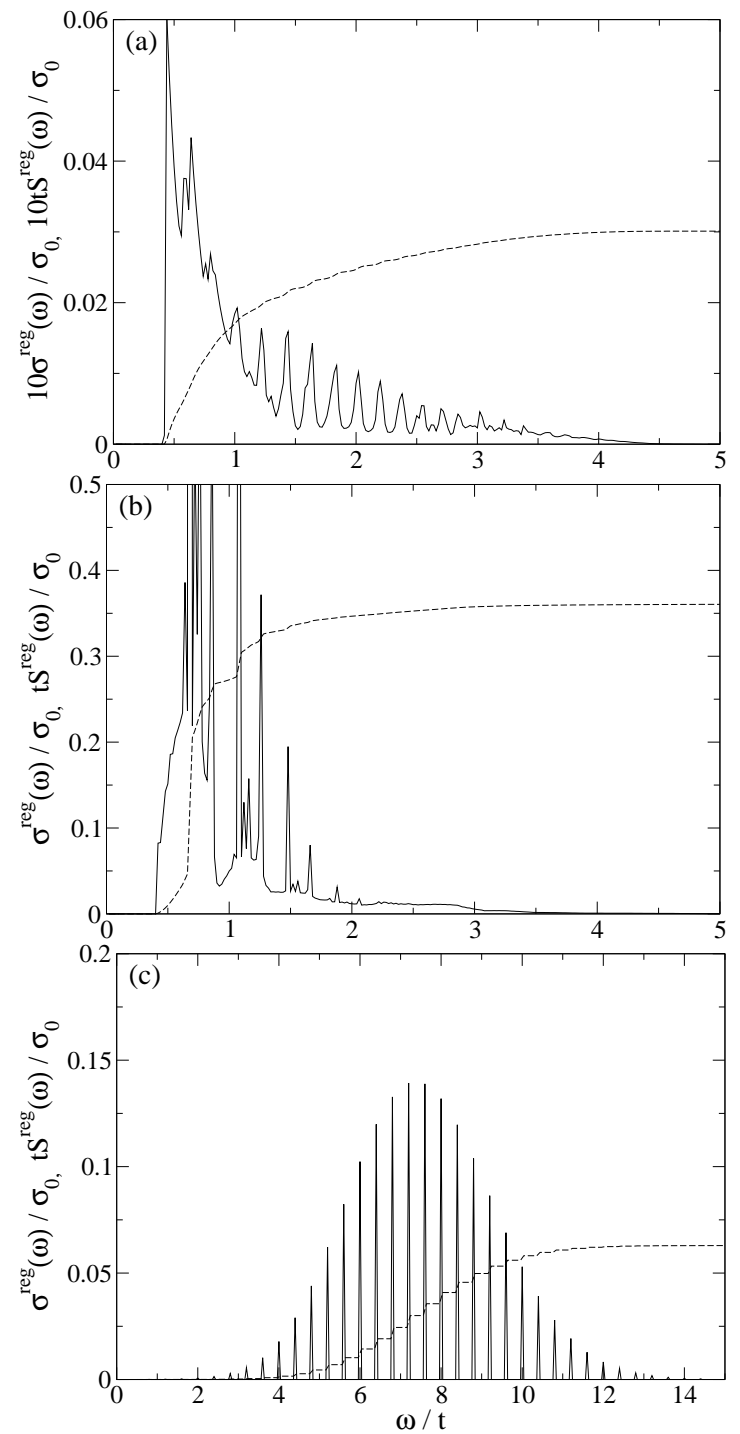

Figure 10. Analytical results for $\sigma^{\mathrm{reg}}(\omega)(-)$ and $S^{\mathrm{reg}}(\omega)(--)^{-}$) from the IC [(a),(b)] respectively SC [(c)] approximation. Here $\omega_{0} / t=0.4, n=0.4$, and (a) $E_{\mathrm{P}} / t=0.1$ ( $R=1.5)$, (b) $E_{\mathrm{P}} / t=2(R=1.3)$ and (c) $E_{\mathrm{P}} / t=4$.

The evolution of $S^{\text {reg }}$ with increasing $E_{\mathrm{P}}$ is similar to that observed in the one-electron case (figure 3(a)), with a maximum in the IC regime. Whereas for weak coupling transport is mainly coherent (as reflected by a large Drude weight $t \mathcal{D} / \sigma_{0} \approx 0.61 \gg t S^{\mathrm{reg}} / \sigma_{0}$ ), $S^{\mathrm{reg}}$ increases noticeably for $E_{\mathrm{P}} / t=2$ owing to enhanced incoherent scattering. For strong coupling, since $\mathcal{D} \rightarrow 0$, the reduced kinetic energy suppresses $S^{\text {reg }}$ via the $\mathrm{f}$-sum rule.

Density dependence at intermediate coupling We finally examine the density dependence of the optical response in the IC regime. To make a connection with previous work [16], we take $\omega_{0} / t=0.4$ and $E_{\mathrm{P}} / t=2$. Figure 11 depicts results for $\sigma^{\mathrm{reg}}(\omega)$ for $n=0.1$ and $n=0.3$, 

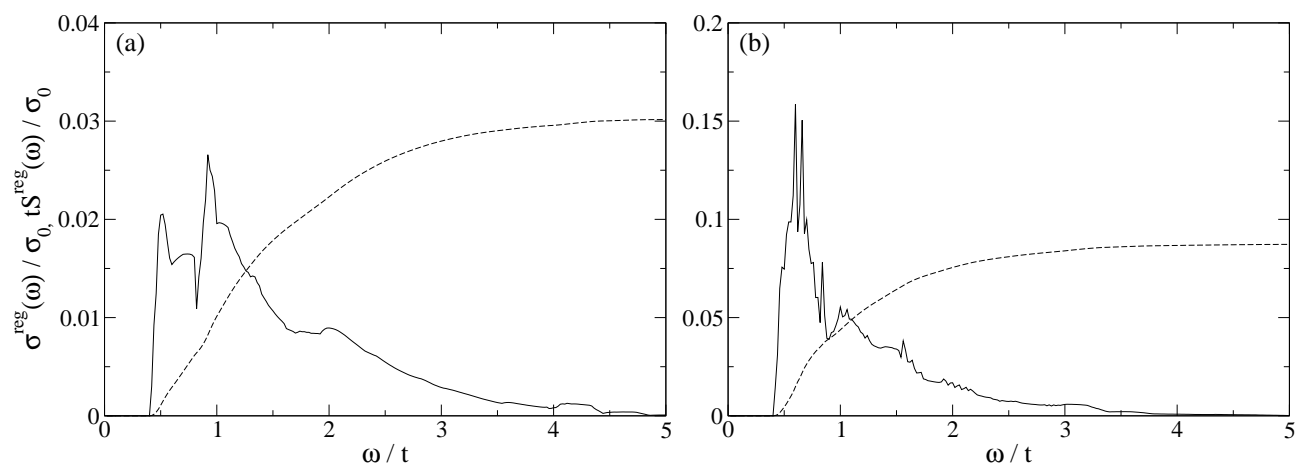

Figure 11. Analytical results for $\sigma^{\mathrm{reg}}(\omega)$ (-) and $S^{\mathrm{reg}}(\omega)$ (- - -) from the IC approximation. Here $\omega_{0} / t=0.4, E_{\mathrm{P}} / t=2.0$, (a) $n=0.1(R=2.1)$ and (b) $n=0.3$ $(R=1.5)$.

whereas the case $n=0.4$ is reported in figure 10 (b).

The low-density case $n=0.1$ has already been shown in figure $5(\mathrm{~b})$, and results are presented without rescaling in figure 11(a) for reference. Compared to $n=0.3$ (figure 11(b)), we observe a transfer of spectral weight from high to low frequencies, causing a strong reduction of the broad high-energy hump. This trend continues upon increasing $n$ even further, as can be seen from figure 10 (b). Besides, the continuous increase of $S^{\text {reg }}$ with increasing $n$ is similar to the WC case depicted in figure 4(a).

These features of the optical conductivity suggest that the increase of the kinetic energy with $n$, as shown in table 1, has a more profound origin than the mere increase of the charge carrier density. In this connection, it is necessary to discuss the dependence of the "optimal" polaron radius $R$ on density $n$. The values reported in the caption of figures 10 and 11 illustrate that within our variational approach, $R$ decreases with increasing carrier density, i.e., $R=2.1$ for $n=0.1, R=1.5$ for $n=0.3$, and $R=1.3$ for $n=0.4$. This seems to be at odds with the crossover from polaronic behaviour at small $n$ to metal-like behaviour at larger $n$ observed numerically [7,8], since a decrease of $R$ with increasing $E_{\mathrm{P}}$ at fixed $n$ generally enhances the polaronic character of the spectra [17].

Whereas the dependence of $\varepsilon_{\text {kin }}$ on the coupling strength at fixed $n$ may be interpreted as the crossover between the small-polaron and large-polaron regime, the concentration dependence of $\varepsilon_{\text {kin }}$ seems to be more involved [7,8]. Indeed, according to the variational treatment of the IC case, the concentration dependence of the kinetic energy and optical conductivity is to be explained by the $n$-dependence of the parameters $\gamma, \bar{\gamma}, \eta$ defined by equations (5),(6). These parameters are determined, using the variational variables $R$, by the energy balance of the Hartree energy of polarons and the energy of the lattice deformation background [17], which at $\lambda=1$ may be quite significant. Although the optimal $R$ increases with decreasing $n$, this energy balance leads to an increase of kinetic energy with increasing $n$, as the resulting increase of the lattice deformation is sufficient to enhance the mobility of charge carriers.

This picture may be supplemented and improved by a more detailed insight provided by the electron-lattice and electron-electron correlations calculated numerically in [8]. According to these results, the increase of the carrier concentration is accompanied by the short-range development of the charge-density wave, which is connected with the spreading of the inhomogeneous lattice deformation about the centre of the polaron. Consequently, the analytical calculations based on the Hartree energy — taking into account only the averaged 
energy of the lattice deformation background - cannot describe these features revealed by numerical calculations. Nevertheless, the optical spectra turn out to reproduce the main features found numerically $[8,16]$, and the dependence of the total energy on $n$ is in good agreement with exact data [8].

\section{Discussion and conclusions}

The results of section 4.2 suggest that the analytical approach captures the main features of polaronic systems with finite carrier density. Therefore, it is interesting to relate our findings to the polaronic excitations observed in manganites, for which no general theory is available. To be more specific, we consider results for thin films of $\mathrm{La}_{2 / 3} \mathrm{Sr}_{1 / 3} \mathrm{MnO}_{3}$ (LSMO) and $\mathrm{La}_{2 / 3} \mathrm{Ca}_{1 / 3} \mathrm{MnO}_{3}$ (LCMO) containing a characteristic mid-infrared peak usually assigned to polarons [5]. The shape and position of this peak differs substantially for the two materials, and has been interpreted in terms of large and small polarons, respectively [5,44]. Apart from the shift of the maximum in $\sigma^{\mathrm{reg}}(\omega)$ to higher energies when going from LSMO to LCMO, the peak is highly asymmetric in LSMO — with a sharp onset just below the maximum and a high-energy tail—and much more symmetric in LCMO.

In small-polaron theory, the maximum in $\sigma^{\mathrm{reg}}(\omega)$ occurs at $2 E_{\mathrm{P}}$ [40], so that experiments can in principle be used to determine $E_{\mathrm{P}}$. However, for small-polaron theory to be applicable, the resulting value has to be consistent with the high-temperature activation energy of the dc conductivity, i.e., $4 E_{\mathrm{a}}=E_{\mathrm{P}}$ [40]. Experimentally, it turns out that this is usually not the case, indicating that small-polaron theory is invalid. This problem has been pointed out in [35], and a many-electron approach valid at intermediate coupling was proposed [3,27]. The basic assumptions are that perovskite LSMO falls into the WC regime, whereas LCMO is characterised by intermediate to strong coupling, in accordance with the Curie temperatures $T_{\mathrm{C}} \approx 370 \mathrm{~K}$ respectively $T_{\mathrm{C}} \approx 270 \mathrm{~K}$ [27], and that the polaronic character of quasiparticles is enhanced in thin films [3]. Hartinger et al [5] compare various theories to their data, concluding that none is able to satisfactorily explain the lineshape of the polaron peak.

Based on the results of our simple analytical theory, we can make the following remarks. The change of shape from asymmetric to symmetric and the position of the maximum is observed when comparing the WC and IC/SC regimes. In particular, the WC approach features a sharp onset and a long high-energy tail, whereas the IC/SC results are reminiscent of the Poisson distribution of phonons. These main features can already be observed in our exact one-electron results in figure 5. Apart from setting the order of magnitude of $\sigma^{\text {reg }}(\omega)$, the many-polaron effects discussed above are of minor importance for weak coupling (as appropriate for LSMO), but renormalise polaronic features toward "metallic" (or more WClike) behaviour in the IC regime [7]. Although polaron effects are enhanced in thin films as used in [5], the discrepancy between $E_{\mathrm{P}}$ as deduced from $\sigma^{\mathrm{reg}}(\omega)$ and $E_{\mathrm{a}}$ for LCMO in [5] suggests that the sample lies near $\left(T_{\mathrm{C}} \approx 240 \mathrm{~K}\right)$ but not in the $\mathrm{SC}$ regime. The true SC case seems to be realised in the layered manganite $\mathrm{La}_{1.2} \mathrm{Sr}_{1.8} \mathrm{Mn}_{2} \mathrm{O}_{7}$, for which angular-resolved photoemission spectra [45] suggest the existence of small polarons even in the ferromagnetic state $[3,46]$.

The present approach correctly predicts coherent states at the Fermi level giving rise to a Drude response, and reproduces standard small-polaron results in the SC limit. This is in contrast to an analytical the treatment of the Holstein double-exchange model [27], which includes the coupling of local and itinerant spins in the manganites. The WC theory for a gas of polarons proposed by in [19] may in principle explain the optical conductivity of LSMO, but it is not clear why long-range electron-phonon interaction is not screened in a dense metallic system. Exact results for the Fröhlich model with one electron have been given 
in [47]. A model of optical absorption based on transitions between ground and excited mixed polaron states, formed by the hybridisation of the narrow small-polaron band, and the wide large-polaron band was presented in $[42,48]$. However, this model is dealing with a number of constants, not explicitly connected with the parameters of the Holstein Hamiltonian, which are to be determined by fitting the experimental absorption curves. Besides, it is not clear how the crossover from the SC to the WC case and the optical absorption in the WC regime can be described in this framework.

To conclude, we have developed an analytical theory for the optical conductivity of finitedensity polaronic systems, based on previous work on the single-particle spectral function. The spectra are surprisingly rich and in satisfactory agreement with exact results. Together with previous numerical results [16], the present work provides a better understanding of the optical conductivity of dense systems with large or small polarons. Finally, it can explain some of the generic polaronic features observed in the manganites, but our electron-phonon model is too simple to make quantitative predictions.

\section{Acknowledgments}

We gratefully acknowledge financial support by the DFG and the Academy of Sciences of the Czech Republic (ASCR) under Grant Nr. 436 TSE 113/33/0-2, and the Austrian Science Fund (FWF) through the Erwin-Schrödinger Grant No J2583. We thank G Wellein for producing the ED data for table 1.

\section{Appendix A.}

The fermion spectral function (of electrons or polarons) can be written as

$$
A(k, \omega)=-\frac{1}{\pi} \frac{\operatorname{Im} \Sigma(k, \omega)}{\left[\omega-\left(\xi_{k}+\eta\right)-\operatorname{Re} \Sigma(k, \omega)\right]^{2}+[\operatorname{Im} \Sigma(k, \omega)]^{2}},
$$

with the fermion self-energy $\Sigma(k, \omega)$, and the free band dispersion of electrons (polarons) $\xi_{k}=-W \cos k\left(\xi_{k}=-\widetilde{W} \cos k\right)$.

For the Hamiltonian (1), the coherent part of the spectrum, $A^{\mathrm{c}}(k, \omega)$-characterised by $\operatorname{Im} \Sigma(k, \omega)=0$-is non-zero for $|\omega|<\omega_{0}$. In this frequency interval,

$$
A^{\mathrm{c}}(k, \omega)=z_{k} \delta\left[\omega-\left(E_{k}+\eta\right)\right],
$$

where $E_{k}$ is the solution of the equation

$$
E_{k}=\xi_{k}+\operatorname{Re} \Sigma\left(k, E_{k}+\eta\right),
$$

and

$$
z_{k}^{-1}=\left|1-\left[\partial_{\omega} \operatorname{Re} \Sigma(k, \omega)\right]_{\omega=E_{k}+\eta}\right| .
$$

Accordingly, the incoherent part of the spectrum, denoted as $A^{\text {ic }}(k, \omega)$, is non-zero for $|\omega|>\omega_{0}$ and can be calculated according to equation (A.1).

The optical conductivity is determined by the electronic spectral function $A_{\mathrm{e}}(k, \omega)$. However, in the $\mathrm{SC}$ case, the correct fermionic quasiparticles are small polarons, and $A_{\mathrm{e}}(k, \omega)$ has to be expressed in terms of the polaronic spectral function $A_{\mathrm{p}}(k, \omega)$ via [17]

$$
\begin{aligned}
A_{\mathrm{e}}(k, \omega)=\mathrm{e}^{-g^{2}} A_{\mathrm{p}}(k, \omega)+\mathrm{e}^{-g^{2}} \frac{1}{N} \sum_{s \geq 1} \frac{\left(g^{2}\right)^{s}}{s !} \\
\quad \times \sum_{k^{\prime}}\left[A_{\mathrm{p}}\left(k^{\prime}, \omega-s \omega_{0}\right) \theta\left(\omega-s \omega_{0}\right)+A_{\mathrm{p}}\left(k^{\prime}, \omega+s \omega_{0}\right) \theta\left(-\omega-s \omega_{0}\right)\right] .
\end{aligned}
$$


For the numerical evaluation of equation (21), we define

$$
\begin{aligned}
A_{\mathrm{e}}^{<}(k, \omega<0)= & \mathrm{e}^{-g^{2}} z_{k} \delta\left[\omega-\left(E_{k}+\eta\right)\right] \theta\left(\omega_{0}+\omega\right)+\mathrm{e}^{-g^{2}} \sum_{s \geq 1} \frac{g^{2 s}}{s !} \frac{1}{N} \\
& \times \sum_{k^{\prime}} z_{k^{\prime}} \delta\left[\omega+s \omega_{0}-\left(E_{k^{\prime}}+\eta\right)\right] \theta\left(-\omega-s \omega_{0}\right) \theta\left[\omega+(s+1) \omega_{0}\right]
\end{aligned}
$$

and

$$
\begin{aligned}
A_{\mathrm{e}}^{>}(k, \omega>0)= & \mathrm{e}^{-g^{2}} z_{k} \delta\left[\omega-\left(E_{k}+\eta\right)\right] \theta\left(\omega_{0}-\omega\right)+\mathrm{e}^{-g^{2}} \sum_{s \geq 1} \frac{g^{2 s}}{s !} \frac{1}{N} \\
& \times \sum_{k^{\prime}} z_{k^{\prime}} \delta\left[\omega-s \omega_{0}-\left(E_{k^{\prime}}+\eta\right)\right] \theta\left(\omega-s \omega_{0}\right) \theta\left[(s+1) \omega_{0}-\omega\right],
\end{aligned}
$$

as well as

$$
\begin{aligned}
A_{\mathrm{e}}^{\mathrm{ic}}(k, \omega \gtrless 0)= & \mathrm{e}^{-g^{2}} A_{\mathrm{p}}^{\mathrm{ic}}(k, \omega) \theta\left( \pm \omega-\omega_{0}\right)+\mathrm{e}^{-g^{2}} \sum_{s \geq 1} \frac{g^{2 s}}{s !} \frac{1}{N} \\
& \times \sum_{k^{\prime}} A_{\mathrm{p}}^{\mathrm{ic}}\left(k^{\prime}, \omega \mp s \omega_{0}\right) \theta\left[ \pm \omega-(s+1) \omega_{0}\right] .
\end{aligned}
$$

Equations (A.5)-(A.8) for the SC case are formally the same in the IC case, with $g$ replaced by $\bar{\gamma} g$ throughout.

The contributions to the integral in equation (23) are then given by

$$
\begin{aligned}
& A=\int_{-\infty}^{0} \mathrm{~d} \omega^{\prime} \theta\left(\omega+\omega^{\prime}\right) A_{\mathrm{e}}^{<}\left(k, \omega^{\prime}\right) A_{\mathrm{e}}^{>}\left(k, \omega^{\prime}+\omega\right), \\
& B=\int_{-\infty}^{0} \mathrm{~d} \omega^{\prime} \theta\left(\omega+\omega^{\prime}\right) A_{\mathrm{e}}^{<}\left(k, \omega^{\prime}\right) A_{\mathrm{e}}^{\mathrm{ic}}\left(k, \omega^{\prime}+\omega\right), \\
& C=\int_{-\infty}^{0} \mathrm{~d} \omega^{\prime} \theta\left(\omega+\omega^{\prime}\right) A_{\mathrm{e}}^{\text {ic }}\left(k, \omega^{\prime}\right) A_{\mathrm{e}}^{>}\left(k, \omega^{\prime}+\omega\right), \\
& D=\int_{-\infty}^{0} \mathrm{~d} \omega^{\prime} \theta\left(\omega+\omega^{\prime}\right) A_{\mathrm{e}}^{\text {ic }}\left(k, \omega^{\prime}\right) A_{\mathrm{e}}^{\text {ic }}\left(k, \omega^{\prime}+\omega\right) .
\end{aligned}
$$

\section{Appendix B.}

The Drude formula for the low-frequency conductivity,

$$
\operatorname{Re} \sigma(\omega)=\frac{\mathcal{D}}{\pi} \frac{\tau}{1+(\omega \tau)^{2}},
$$

gives at $\omega=0$ for the dc conductivity

$$
\sigma(0)=\mathcal{D} \tau / \pi .
$$

Here $\tau$ denotes the relaxation time. In the limit $1 / \tau \rightarrow 0$, we obtain

$$
\operatorname{Re} \sigma(\omega)=\mathcal{D} \delta(\omega) \text {. }
$$

The standard way to deduce the conductivity at $\omega=0$ using the Kubo formula is to take the limit $\omega \rightarrow 0$ of $\operatorname{Re} \sigma(\omega)$ calculated for $\omega>0$ [40]. However, equation (21) yields zero for $0<\omega<\omega_{0}$. To derive the Drude-like singularity (B.3) for the formulation of the f-sum rule, we shall start with a low but non-zero temperature $T$. Moreover, the spectral function in 
equation (21) is assumed to have a finite width—caused by a very weak additional scattering mechanism-leading to

$$
A\left(k, \omega^{\prime}\right)=\frac{\kappa z_{k}}{\pi} \frac{\Delta}{\left[\omega^{\prime}-\left(E_{k}+\eta\right)\right]^{2}+\Delta^{2}},
$$

where $\Delta=(2 \tau)^{-1}$. In the WC approach, $\kappa=1, \eta=-\mu$, whereas in the IC/SC approach $\kappa=\exp \left(-g^{2} \bar{\gamma}^{2}\right)$, and $\eta$ is given by equations (3) respectively (5).

After substitution of equation (20) into equation (8), the limit $\omega \rightarrow 0$ gives

$\lim _{\omega \rightarrow 0} \operatorname{Re} \sigma(\omega)=\sigma(0)=-4 \sigma_{0} \frac{1}{N} \sum_{k}(\sin k)^{2} \int_{-\infty}^{\infty} \mathrm{d} \omega^{\prime}\left[A\left(k, \omega^{\prime}\right)\right]^{2} \mathrm{~d}_{\omega^{\prime}} f\left(\omega^{\prime}\right)$.

The subsequent limit $T \rightarrow 0$ is taken using $\left[\mathrm{d}_{\omega^{\prime}} f\left(\omega^{\prime}\right)\right]_{T=0}=-\delta\left(\omega^{\prime}\right)$, which after substitution of equation (B.4) into equation (B.5) yields

$$
\lim _{T \rightarrow 0} \sigma(0)=\frac{4 \kappa^{2} \sigma_{0}}{\pi^{2}} \frac{1}{N} \sum_{k}(\sin k)^{2} z_{k}^{2} \frac{\Delta^{2}}{\left[\left(E_{k}+\eta\right)^{2}+\Delta^{2}\right]^{2}} .
$$

Converting the sum over $k$ into an integral over $E_{k}$ we obtain

$$
\sigma(0)=\frac{4 \kappa^{2} \sigma_{0}}{\pi^{3}} \int_{\left\{E_{k}\right\}} \mathrm{d} E_{k}(\sin k)^{2} \frac{z_{k}^{2}}{\partial_{k} E_{k}} \frac{\Delta^{2}}{\left[\left(E_{k}+\eta\right)^{2}+\Delta^{2}\right]^{2}},
$$

where the integration is over the energy range of the coherent part of the spectrum. However, considering $\Delta \rightarrow 0$, the integrated function is non-zero only in a narrow interval around $E_{k}+\eta=0$, i.e., for $k \approx k_{\mathrm{F}}$. Assuming the slowly varying part of the integrand to be constant, the integral reduces to

$$
\int_{-\infty}^{\infty} \mathrm{d} E_{k} \frac{\Delta^{2}}{\left[\left(E_{k}+\eta\right)^{2}+\Delta^{2}\right]^{2}}=\frac{\pi}{2 \Delta}=\pi \tau
$$

Comparison with equation (B.2) then yields

$$
\sigma(0)=\frac{4 \kappa^{2} \sigma_{0}}{\pi^{2}}\left(\sin k_{\mathrm{F}}\right)^{2}\left[\frac{z_{k}^{2}}{\partial_{k} E_{k}}\right]_{k=k_{\mathrm{F}}} \tau \equiv \mathcal{D} \tau / \pi
$$

According to equation (B.3), for $1 / \tau \rightarrow 0$, the Drude singularity takes the form

$$
[\operatorname{Re} \sigma(\omega)]_{D}=\mathcal{D} \delta(\omega)=\frac{4 \kappa^{2} \sigma_{0}}{\pi}\left(\sin k_{\mathrm{F}}\right)^{2}\left[\frac{z_{k}^{2}}{\partial_{k} E_{k}}\right]_{k=k_{\mathrm{F}}} \delta(\omega) .
$$

In particular, in the WC approximation with $\bar{\gamma}=0$ in equation (25) and $\kappa=1$,

$$
\mathcal{D}=2 t e^{2} a z_{k} \sin k_{\mathrm{F}}
$$

To check the above considerations, we briefly examine the free-electron case. In the latter, $S^{\text {reg }} \rightarrow 0$, and the kinetic energy per site is given as

$$
\varepsilon_{\text {kin }}=\frac{1}{N} \sum_{|k|<k_{\mathrm{F}}}(-2 t \cos k)=-\frac{1}{\pi} 2 t \sin k_{\mathrm{F}} .
$$

Since $z_{k}=1$, equation (34) is fulfilled.

In the SC limit $g^{2} \gg 1$, the leading term of $\mathcal{D}$ is

$$
\mathcal{D} \approx \widetilde{W} e^{2} a \sin k_{\mathrm{F}},
$$

having the form of the Drude term of free carriers with half-bandwidth $\widetilde{W}$.

Comparing this estimate of $\mathcal{D}$ to the integrated regular part of the conductivity $S^{\text {reg }}$, we find that the Drude part is negligible in the SC limit, so that the kinetic energy is determined by 
$S^{\text {reg }}$ according to the f-sum rule (34). To demonstrate this, we shall not use equation (24), but instead start with $[\operatorname{Re} \sigma(\omega)]_{A}$ following from equations (A.6),(A.7), (A.9) and carry out the integration over $\omega$ before integrating over $k$. The dominating part of $[\operatorname{Re} \sigma(\omega)]_{A}$ for estimating $S^{\text {reg }}$ in the SC limit (corresponding to the contributions (A3) in figure 2) reads

$$
\begin{aligned}
\operatorname{Re} \sigma(\omega)=\frac{2 \sigma_{0}}{\pi^{2} \omega} \mathrm{e}^{-2 g^{2}} \sum_{s \geq 1} \frac{\left(2 g^{2}\right)^{s}}{s !} \int_{0}^{\pi} \mathrm{d} k^{\prime} z_{k^{\prime}} \theta\left(-E_{k^{\prime}}-\eta\right) \\
\quad \times \int_{0}^{\pi} \mathrm{d} k^{\prime \prime} z_{k^{\prime \prime}} \theta\left(E_{k^{\prime \prime}}+\eta\right) \delta\left(\omega-s \omega_{0}-E_{k^{\prime \prime}}+E_{k^{\prime}}\right),
\end{aligned}
$$

where we assumed $\left|E_{k}\right|<\omega_{0}$ for all $E_{k}$ in the SC limit. The integration over $\omega$ gives

$$
\begin{aligned}
S^{\mathrm{reg}}=\frac{2 \sigma_{0}}{\pi^{2}} \mathrm{e}^{-2 g^{2}} & \sum_{s \geq 1} \frac{\left(2 g^{2}\right)^{s}}{s !} \int_{0}^{\pi} \mathrm{d} k^{\prime} z_{k^{\prime}} \theta\left(-E_{k^{\prime}}-\eta\right) \\
& \times \int_{0}^{\pi} \mathrm{d} k^{\prime \prime} z_{k^{\prime \prime}} \theta\left(E_{k^{\prime \prime}}+\eta\right) \frac{1}{s \omega_{0}-E_{k^{\prime \prime}}+E_{k^{\prime}}} .
\end{aligned}
$$

To estimate $S^{\text {reg }}$, we set $z_{k^{\prime}}, z_{k^{\prime \prime}}=1$ and assume $E_{k^{\prime}}, E_{k^{\prime \prime}} \ll s \omega_{0}$. Accordingly, the rational function in equation (B.15) is replaced by $1 / s \omega_{0}$ and the integrals over $k^{\prime}, k^{\prime \prime}$ simply give $k_{\mathrm{F}}$ and $\pi-k_{\mathrm{F}}$, respectively. The Fermi wavevector corresponding to the band population at $T=0$ is $k_{\mathrm{F}}=n \pi$. The resulting estimate following from equation (B.15) is thus given as

$$
S^{\mathrm{reg}}=\frac{2 \sigma_{0}}{\omega_{0}} n(1-n) \sum_{s \geq 1} \mathrm{e}^{-2 g^{2}} \frac{\left(2 g^{2}\right)^{s}}{s !} \frac{1}{s}=\frac{2 \sigma_{0}}{\omega_{0}} n(1-n)\left\langle\frac{1}{s}\right\rangle_{2 g^{2}}
$$

The brackets symbolise averaging with respect to the Poisson distribution with the parameter $2 g^{2}$, and the concentration dependence of equation (B.16) is in agreement with standard small-polaron theory based on the atomic-limit approximation [40].

Comparison of equation (B.16) and equation (B.13) reveals that the Drude term in the f-sum rule (34) is negligible in the SC limit, so that $\varepsilon_{\text {kin }}$ is proportional to $S^{\text {reg }}$,

$$
\varepsilon_{\text {kin }}=-\frac{(2 t)^{2}}{\omega_{0}} n(1-n)\left\langle\frac{1}{s}\right\rangle_{2 g^{2}}
$$

To make another check of the latter result, we compare equation (B.17) to the kinetic energy of one polaron at the bottom of the band, obtained from equation (A.3) as $t\left[\partial_{k} E_{k}\right]_{k=0}$ in [49]. Keeping only the leading term with respect to $\mathrm{e}^{-g^{2}}$, setting $z_{k}=1$ and neglecting $E_{k}$ in comparison to $\omega_{0}$ as above, we obtain

$$
E_{\text {kin }}=-\frac{(2 t)^{2}}{\omega_{0}}\left\langle\frac{1}{s}\right\rangle_{2 g^{2}} .
$$

Assuming $n \ll 1, \varepsilon_{\text {kin }}=n E_{\text {kin }}$, in accordance with equation (B.17).

\section{References}

[1] P Calvani, P Dore, S Lupi, A Paolone, P Maselli, P Giura, B Ruczicka, S-W Cheong, and W Sadowski, J. Supercond. 10, 293 (1997);

K A Müller, J. Supercond. 12, 3 (1999).

[2] E K H Salje, A S Alexandrov, and W Y Liang, Polarons and Bipolarons in High Temperature Superconductors and Related Materials (Cambridge Univ. Press, Cambridge, 1995).

[3] M Hohenadler and D M Edwards, J. Phys.: Condens. Matter 14, 2547 (2002).

[4] D M Edwards, Adv. Phys. 51, 1259 (2002).

[5] C Hartinger, F Mayr, A Loidl, and T Kopp, Phys. Rev. B 73, 024408 (2006). 
[6] M Capone, M Grilli, and W Stephan, Eur. Phys. J. B 11, 551 (1999).

[7] M Hohenadler, D Neuber, W von der Linden, G Wellein, J Loos, and H Fehske, Phys. Rev. B 71, 245111 (2005).

[8] M Hohenadler, G Hager, G Wellein, and H Fehske, J. Phys.: Condens. Matter (in press).

[9] T Holstein, Ann. Phys. (N.Y.) 8, 325; 8, 343 (1959).

[10] H Fröhlich, Adv. Phys. 3, 325 (1954).

[11] G Schubert, G Wellein, A Weiße A Alvermann, and H Fehske, Phys. Rev. B 72, 104304 (2005).

[12] Y. Y. Suzuki, P. Pincus, and A. J. Heeger, Phys. Rev. B 44, 7127 (1991).

[13] H Fehske, D Ihle, J Loos, U Trapper, and H Büttner, Z. Phys. B 94, 91 (1994).

[14] C A Perroni, G De Filippis, V Cataudella, and G Iadonisi, Phys. Rev. B 64, 144302 (2001).

[15] H Fehske, G Wellein, G Hager, A Weiße, and A R Bishop, Phys. Rev. B 69, 165115 (2004).

[16] G Wellein, A R Bishop, M Hohenadler, G Schubert, and H Fehske, Physica B 378-380, 281 (2006).

[17] J Loos, M Hohenadler, and H Fehske, J. Phys.: Condens. Matter 18, 2453 (2006).

[18] J Loos, M Hohenadler, A Alvermann, and H Fehske, J. Phys.: Condens. Matter 18, 7299 (2006).

[19] J Tempere and J T Devreese, Phys. Rev. B 64, 104504 (2001).

[20] V L Gurevich, I E Lang, and Yu A Firsov, Fiz. Tverd. Tela 4, 1252 (1962), [Sov. Phys.-Solid State 4, 918 (1962)].

[21] A S Alexandrov and N Mott, Polarons \& Bipolarons (World Scientific, Singapore, 1995).

[22] H Fehske, A Alvermann, M Hohenadler, and G Wellein, in Polarons in Bulk Materials and Systems with Reduced Dimensionality, Proc. Int. School of Physics "Enrico Fermi", Course CLXI, edited by G Iadonisi, J Ranninger, and G De Filippis (IOS Press, Amsterdam, Oxford, Tokio, Washington DC, 2006), pp. 285-296.

[23] S Sykora, A Hübsch, K W Becker, G Wellein, and H Fehske, Phys. Rev. B 71, 045112 (2005).

[24] C E Creffield, G Sangiovanni, and M Capone, Eur. Phys. J. B 44, 175 (2005).

[25] M Hohenadler, G Wellein, A R Bishop, A Alvermann, and H Fehske, Phys. Rev. B 73, 245120 (2006).

[26] S Sykora, A Hübsch, and K W Becker, Eur. Phys. J B 51, 181 (2006).

[27] A C M Green, Phys. Rev. B 63, 205110 (2001).

[28] I G Lang and Y A Firsov, Zh. Eksp. Teor. Fiz. 43, 1843 (1962), [Sov. Phys. JETP 16, 1301 (1962)].

[29] D N Zubarev, Nonequilibrium statistical thermodynamics (Plenum Press, New York, 1974).

[30] G Rickayzen, Green's Functions and Condensed Matter (Academic Press, Inc., London, 1984).

[31] T Pruschke, M Jarrell, and J K Freericks, Adv. Phys. 44, 187 (1995).

[32] P F Maldague, Phys. Rev. B 16, 2437 (1977).

[33] D Baeriswyl, C Gros, and T M Rice, Phys. Rev. B 35, 8391 (1987).

[34] D Sénéchal, D Perez, and D Plouffe, Phys. Rev. B 66, 075129 (2002).

[35] W Kohn, Phys. Rev. 133, A171 (1964).

[36] J Bonča, S A Trugman, and I Batistic, Phys. Rev. B 60, 1633 (1999).

[37] A Weiße, G Wellein, A Alvermann, and H Fehske, Rev. Mod. Phys. 78, 275 (2006).

[38] H Fehske and S A Trugman, in Polarons in Advanced Materials, edited by A S Alexandrov (Canopus Publishing and Springer Verlag GmbH, Bath (UK), 2007).

[39] D Emin, Phys. Rev. B 48, 13691 (1993).

[40] G D Mahan, Many-Particle Physics, 2nd ed. (Plenum Press, New York, 1990).

[41] A S Alexandrov, V V Kabanov, and D K Ray, Phys. Rev. B 49, 9915 (1994).

[42] D M Eagles, J. Phys. C: Solid State Phys. 17, 637 (1984).

[43] J Loos and J Straka, Czech J. Phys. 93, 316 (1989).

[44] K. H. Kim, J. H. Jung, and T. W. Noh, Phys. Rev. Lett. 81, 1517 (1998).

[45] D S Dessau, T Saitoh, C-H Park, Z-X Shen, P Villella, N Hamada, Y Moritomo, and Y Tokura, Phys. Rev. Lett. 81, $192(1998)$.

[46] A S Alexandrov and A M Bratkovsky, J. Phys.: Condens. Matter 11, L531 (1999).

[47] A S Mishchenko, N Nagaosa, N V Prokof'ev, A Sakamoto, and B V Svistunov, Phys. Rev. Lett. 91, 236401 (2003).

[48] D M Eagles, J. Phys. C: Solid State Phys. 17, 655 (1984).

[49] H Fehske, J Loos, and G Wellein, Phys. Rev. B 61, 8016 (2000). 